\title{
Monopoles and Baker Functions
}

\author{
N. Ercolani ${ }^{1 \star}$ and A. Sinha ${ }^{2}$ \\ ${ }^{1}$ Department of Mathematics, University of Arizona, Tucson, AZ 85721, USA \\ ${ }^{2}$ Department of Mathematics, The Ohio State University, Columbus, OH 43210, USA
}

\begin{abstract}
The work in this paper pertains to the solutions of Nahm's equations, which arise in the Atiyah-Drinfield-Hitchin-Manin-Nahm construction of solutions to the Bogomol'nyi equations for static monopoles. This paper provides an explicit construction of the solution of Nahm's equations which satisfy regularity and reality conditions. The Lax form of Nahm's equations is reduced to a standard eigenvalue problem by a special gauge transformation. These equations may then be solved by the method of Baker-Krichever. This leads to a compact representation of the solutions of Nahm's equations. The regularity condition is shown to be related to the monodromy of the gauge reduced linear operator. Hitchin showed that the solutions of Nahm's equations can be characterized by an algebraic curve and some data on that curve. Here, this characterization reduces to a transcendental equation involving certain loop integrals of a meromorphic differential. Donaldson coordinatized the moduli space of $k$-monopoles by a class of rational maps from the Riemann sphere to itself. The data of a Baker function is equivalent to this map. This method gives an "apriori" construction of the (known) two monopole solutions. We also give a generalization of the two monopole solution to a class of elliptic solutions of arbitrary charge. These solutions correspond to reducible curves with elliptic components and the associated Donaldson rational function has a simple partial fraction expansion.
\end{abstract}

\section{Introduction}

The work in this paper pertains to the solutions of the $S U(2)$-Bogomol'nyi [1] equations. These solutions are called monopoles and have been the subject of extensive analysis by Nahm [2], Atiyah and Hitchin [3], and Donaldson

\footnotetext{
* Supported in part by the National Science Foundation, Grant Number DMS-8701318 and the Arizona Center for Mathematical Sciences, sponsored by AFOSR Contract F49620-86-C0130 with the University Research Initiative Program at the University of Arizona
} 
[4] among others [5]. In particular we concentrate on the algebro-geometric formulation of Hitchin $[6,7]$. This approach classifies monopoles in terms of solutions to a periodic Lax equation (Nahm's equations I.1) built from an algebraic curve.

For completeness, we begin with a brief historical review. The $S U(2)-$ Bogomol'nyi equations are ${ }^{1}$

$$
B_{i} \equiv \frac{1}{2} \varepsilon_{i j k} F_{j k}=D_{i} \Phi .
$$

Here $A_{i}, i=1,2,3$ and $\Phi$ are $2 \times 2$ skew-hermitian zero-trace matrix (i.e. $s u(2)$ valued) functions of $x_{1}, x_{2}, x_{3}$; and $F_{i j}=\partial_{i} A_{j}-\partial_{j} A_{i}+\left[A_{i}, A_{j}\right]$ and $D_{i} \Phi=\partial_{i} \Phi+$ $\left[A_{i}, \Phi\right], \partial_{j}=\partial / \partial x_{j}, i, j=1,2,3 . A_{i}$ are the $(S U(2))$ gauge potentials, $F_{i j}$ are the field strengths, and $\Phi$ is a scalar field, called the Higgs field, transforming via the adjoint representation of $S U(2)$. Geometrically $A=A_{i} d x_{i}$ should be viewed as a connection on an $S U(2)$ principal fiber bundle over $\mathbb{R}^{3}$ and $F=F_{i j} d x_{i} \wedge d x_{j}$ is the associated curvature and $\Phi$ is a section of the adjoint bundle. The $C^{\infty}$ solutions of (1) with the boundary condition,

$$
\|\Phi\|=\frac{1}{2} \operatorname{tr} \Phi^{*} \Phi \rightarrow 1
$$

as $r=\sqrt{x_{1}^{2}+x_{2}^{2}+x_{3}^{2}} \rightarrow \infty$, gives static, localized solutions with "minimum" energy of the field equations of a spontaneously broken gauge theory in the so-called Prasad-Sommerfield [1] limit. Note that Eq. (1) is invariant under the gauge transformation

$$
A_{i} \rightarrow g^{-1} A_{i} g+g^{-1} \partial_{i} g, \quad \Phi \rightarrow g^{-1} \Phi g,
$$

where $g\left(x_{1}, x_{2}, x_{3}\right) \in S U(2)$.

The boundary condition (2) gives a map of the "sphere at $\infty$ in space" into the field values $\Phi$ satisfying $\|\Phi\|=1$, or, topologically, $S^{2}$. Thus the solutions of (1) are classified by an element of $\pi_{2}\left(S^{2}\right)=\mathbb{Z}$ provided by this map, i.e. these solutions are characterized by an integer $k$, called the magnetic charge or topological charge of the solution. Solutions of the Bogomol'nyi equation which minimize the energy, for a given magnetic charge $k$, are called monopoles. These solutions depend on $4 k-1$ parameters.

The Bogomol'nyi equations can be viewed as time $\left(x_{0}\right)$ independent solutions of the self-duality equations $F=* F$ in $\mathbb{R}^{4}$ with coordinates $x_{0}, x_{1}, x_{2}, x_{3}$, the gauge fields $A_{1}, A_{2}, A_{3}$ being identified with gauge fields $A_{1}, A_{2}, A_{3}$ in $\mathbb{R}^{3}$ and the Higgs field $\Phi$ being identified with $A_{0}$. Nahm [2] used this formal identification to adapt the ADHM [8] solution of the self-duality problem to the Bogomol'nyi equation. This approach can be summarized as follows: Let $T_{i}(z), i=1,2,3$, where $z$ is an auxiliary variable, be $k \times k$ matrices, satisfying the Nahm equations

$$
\frac{d T_{i}}{d z}=\frac{1}{2} \varepsilon_{i j k}\left[T_{j}, T_{k}\right]
$$

and Hitchin's [7] conditions

\footnotetext{
1 The summation convention is adopted in this section.
} 
C1 $T_{i}^{*}(z)=-T_{i}(z)$,

C2 $T_{i}(z)=-\bar{T}_{i}(-z)$,

C3 $T_{i}(z)$ are analytic functions of $z \in(-1,1)$ and have simple poles at $z= \pm 1$,

C4 The residues of $T_{i}(z)$ at $z= \pm 1$ give an irreducible $k$-dimensional representation of $s u(2)$.

The Nahm (4) equations can be rewritten [7] as a Lax equation, by defining

$$
\begin{gathered}
A=A_{-1} \zeta^{-1}+A_{0}+A_{1} \zeta, \\
A_{+}=\frac{1}{2} A_{0}+A_{1} \zeta, \\
A_{-1}=T_{1}+i T_{2}, \quad A_{0}=-2 i T_{3}, \quad A_{1}=T_{1}-i T_{2},
\end{gathered}
$$

then Eq. (4) is equivalent to

$$
\frac{d A}{d z}=\left[A, A_{+}\right]
$$

It was shown by Hitchin that under these conditions, the operator

$$
\Delta=\left(i \frac{d}{d z}+x_{0}\right)+e_{j}\left(x_{j}+i T_{j}\right),
$$

where $e_{j}, j=1,2,3$ are the imaginary quaternions, has quaternionic index 1 . Thus let $v$, a $k$-component vector of quaternions, be the unique solution to

$$
\Delta^{+} v=0
$$

and

$$
\int_{-1}^{1} v^{+} v=1
$$

Here ${ }^{+}$means transposed and quaternionic conjugate. Note that $v$ has the form

$$
v\left(z, x_{0}, x_{1}, x_{2}, x_{3}\right)=e^{i x_{0} z} N\left(z, x_{1}, x_{2}, x_{3}\right) \text {. }
$$

Then, the solution to (1) with (2) is given by

$$
\Phi\left(x_{1}, x_{2}, x_{3}\right)=\int_{-1}^{1} z N^{+} N d z, \quad A_{i}\left(x_{1}, x_{2}, x_{3}\right)=\int_{-1}^{1} N^{+} \partial_{i} N d z, \quad i=1,2,3 .
$$

This solution has magnetic charge $k$, i.e. this gives a $k$-monopole solution. Moreover, it was shown by Hitchin [7] that all solutions are obtained this way.

Our contribution, in this paper, is the construction of explicit solutions of Nahm's equations which satisfy the regularity and reality conditions required for the physical monopoles. We begin with Hitchin's approach to solving Nahm's equations. To a monopole he associates a so-called spectral curve (i.e. a Riemann surface) together with the data of a line bundle on this curve. This is analogous to the direct scattering procedure for $\mathrm{KdV}$ solitons in which a soliton is associated to certain spectral variables that evolve simply in time. Here, the bundle evolves simply, in the Nahm "time" $z$, on the Jacobian (a torus) of the curve.

In order to explicitly solve Nahm's equations one needs to invert Hitchin's map by an "inverse scattering" procedure. There is a standard inverse procedure 
for solving Lax type equations which goes back to Baker [9], but its more recent formulation is due to Krichever [10]. Unforturnately, these methods are not directly applicable to the Nahm-Hitchin equation (6). The difficulty is that Hitchin's covariant derivative

$$
\frac{d}{d z}+\frac{1}{2} A_{0}(z)+A_{1}(z) \zeta
$$

does not have the form of a standard eigenvalue problem because of the $z$ dependence of $A_{1}$.

The first result is to observe that "gauge transformation" by $C(z)$ which satisfies

$$
\frac{d C}{d z}=\frac{1}{2} A_{0} C
$$

reduces (10) to an eigenvalue problem:

$$
\frac{d}{d z}+Q_{0}(z)+Q_{1}(0) \zeta
$$

and the reduced Nahm equations become:

$$
\frac{d Q}{d z}=\left[Q, Q_{+}\right]
$$

where $Q_{i}=C^{-1} A_{i} C\left(Q_{1}=A_{1}(0)\right), Q=\zeta^{-1} Q_{-1}+Q_{0}+\zeta Q_{1}$ and $Q_{+}=Q_{0}+\zeta Q_{1}$.

This reduction may be explained geometrically. Hitchin [7] has observed that there is a hierarchy of equations, like Nahm's, which correspond to other flows on the Jacobian of the spectral curve. He further remarks that these flows do not commute on the space of Nahm matrices. However, they do commute abstractly on the Jacobian as shown and explained by Griffiths [11]. This is due to the fact that the connection determined by (10), which defines the horizontal lift of the Jacobian flows into skew-hermitian matrices, has non-vanishing curvature which obstructs the commutation of flows. Equation (11b), on the other hand, may be regarded as the first in a hierarchy of commuting flows which are Hamiltonian with respect to the natural Lie-Poisson bracket on the coadjoint orbit of a Kac-Moody algebra [12]. The relation between these two hierarchies and possible Hamiltonian structures for Nahm's equations are interesting topics for future consideration, but will not be treated further here.

H. F. Baker showed how to systematically represent a function, the Baker function, on a Riemann surface, having prescribed transcendental singularities, in terms of integrals of abelian differentials. Krichever applied these ideas to construct the so-called "finite gap" Schrödinger eigenfunctions by matching their asymptotics for large eigenvalues to the transcendental singularities of a Baker function. We apply this method to (11) and construct $Q_{0}$ in terms of theta functions for the spectral curve:

$$
\begin{aligned}
\left(Q_{0}\right)_{i j}= & -\left(\rho_{i}-\rho_{j}\right) c_{i j} \exp \left\{\left(v_{j}-v_{i}\right) z\right\} \\
& \cdot \frac{\Theta\left(W\left(\infty_{j}\right)-\vec{\kappa} z-W\left(\Delta_{i}\right)-K\right) \Theta\left(W\left(\infty_{i}\right)-W\left(\Delta_{i}\right)-K\right)}{\Theta\left(W\left(\infty_{j}\right)-W\left(\Delta_{i}\right)-K\right) \Theta\left(W\left(\infty_{i}\right)-\vec{\kappa} z-W\left(\Delta_{i}\right)-K\right)},
\end{aligned}
$$


where $\Theta$ is the Riemann's theta function (a Fourier series, see Eq. (II.15)), $W$ is Abel's map (see Eq. (II.14)), $\infty_{j}$ are the transcendental singularities of the Baker eigenfunctions for (11a) and $\Delta_{i}$ is a certain divisor on the curve. The frequency vector $\vec{\kappa}$, the normalization constants $\rho_{i}, v_{i}, c_{i j}$ and the Riemann constant $K$ can be explicitly computed from the curve and the data of Hitchin's line bundle.

The organization of this paper is that in Sect. I we derive the reduced equations (11) which are solved in Sect. II with Baker functions. Section II also shows from the solution (12) how Hitchin's gauge invariant formulation of the boundary conditions is satisfied, and how the boundary conditions in Nahm's formulation are met. Although this may seem redundant, it has led to an elegant formulation, Theorem I.1, of the monopole non-singularity condition for Eq. (11b). Namely, the operator (11a) has regular singular points with integer exponents of singularity. The non-singularity condition becomes the requirement that, at singular points, this operator has scalar monodromy; i.e. the solutions of the associated homogeneous equation have no logarithm in their expansion at a singularity. This formulation may be useful in the construction, either analytical or numerical, of a physical $k$-monopole.

In Sect. III, Donaldson's rational map, which encodes the moduli space of $k$-monopoles, is constructed in terms of Baker data. Section IV spells out completely formulas like (12), and uses these for the construction of the two monopole solution. Also presented here is a class of elliptic solutions of arbitrary charge which correspond to reducible spectral curves with elliptic components, and, one finds an expression for Donaldson's map in this case:

$$
f(\lambda)=\left(\frac{-1}{\mathbf{K}}\right)^{k-1} \sum_{j=1}^{k} \frac{\left(\begin{array}{l}
k-1 \\
j-1
\end{array}\right)}{\lambda-i(k+1-2 j) \mathbf{K} / 2},
$$

where $\mathbf{K}$ is the complete elliptic integral of the first kind.

It is assumed, except in Sect. IV, that the spectral curve is non-singular.

To satisfy the boundary conditions these curves must satisfy a set of quantization conditions:

$$
\oint_{b_{j}} \gamma_{\infty}=\pi i m_{j} \text { for some } m_{j} \in \mathbb{Z},
$$

where $\gamma_{\infty}$ is a differential of the second kind canonically associated to the spectral curve (see (II.13)) and $b_{j}$ are the normalized $b$-homology cycles on the curve. These are transcendental equations on the moduli space of curves and it has not yet been proved that these equations have solutions in the domain of non-singular curves. However, this is likely to be true and these results will enable one to study this question perturbatively for 3-monopoles.

Atiyah and Hitchin have studied the dynamics of slowly moving monopoles under the reasonable ansatz that they follow geodesics in the space of static $k$-monopoles. The Baker formulation given here provides the natural ingredients for studying this question through a kind of two-timing perturbation analysis called modulation theory in the spirit of [13-15]. Because of the "periodic" quantization conditions, the effect of slowly moving monopoles on the differential 
$\gamma_{\infty}$ may have some structural similarity with the modulation differentials of a perturbed sine-Gordon equation [15].

\section{Gauge Normalization of Nahm's Equations}

The $k^{\text {th }}$ order Nahm's equations may be written in the Lax form ${ }^{2}$ :

$$
A^{\prime}=\left[A, A_{+}\right] \text {, }
$$

where $A=A_{-1} \zeta^{-1}+A_{0}+A_{1} \zeta$ with $A_{i} \in S l(k, \mathbb{C})$ satisfying the reality conditions ${ }^{3}$

$$
A_{0}^{*}=A_{0}, \quad A_{1}^{*}=-A_{-1} .
$$

$A_{+}=\frac{1}{2} A_{0}+A_{1} \zeta$ and, for later reference, we set $A_{-}=\frac{1}{2} A_{0}+A_{-1} \zeta^{-1}$. Equivalently, one can rewrite (I.1) as the triple

$$
\begin{aligned}
A_{1}^{\prime} & =\frac{1}{2}\left[A_{0}, A_{1}\right], \\
A_{0}^{\prime} & =\left[A_{-1}, A_{1}\right], \\
A_{-1}^{\prime} & =-\frac{1}{2}\left[A_{0}, A_{-1}\right] .
\end{aligned}
$$

We will study solutions of (I.1) subject to the following boundary conditions ${ }^{4}$ on $A_{i}$ :

$A_{i}(z)$ are analytic in $(-1,1)$ and meromorphic near the endpoints, having simple poles at \pm 1 ; i.e., $A_{i}(z)=\frac{a_{i}}{z-1}+R_{i}(z)$, with $R_{i}$ analytic, near $z=1$ and a similar expansion holds near $z=-1$.

The residues $a_{0}, a_{1}$ as operators on $\mathbb{C}^{k}$, have no non-trivial invariant subspaces.

$$
A_{i}(-z)=A_{i}^{t}(z)
$$

The Lax equation (I.1) can be integrated via the spectral theory of the linear differential operator (with parameter $\zeta$ )

$$
D+A_{+}, \quad(D=\partial / \partial z) .
$$

This spectral transform method [10] will be the subject of this and the next section. Remark 1. It follows from Eq. (I.1a) and condition (I.2a) that the residues $a_{0}, a_{-1}$ satisfy the bracket condition

$$
\left[a_{0}, a_{-1}\right]=2 a_{-1} \text {. }
$$

Hence, $a_{-1}$ plays the role of a raising operator and $a_{0}$ has the integer eigenvalues $\{-(k-1),-(k-3), \ldots,(k-3),(k-1)\}$. By examining $(\mathrm{I} .1 \mathrm{~b}, \mathrm{c})$ as well, one can

${ }^{2} A^{\prime}=\partial A / \partial z$ and $[A, B]=A B-B A$

${ }^{3} A^{*}$ denotes the conjugate transpose of $A$ and $A^{t}$ denotes the transpose

${ }^{4}$ The matrices $A_{i}$ presented here differ by a unit translation from those of Hitchin [7] which satisfy $\tilde{A}_{i}(2-z)=\tilde{A}_{i}^{t}(z)$. The relation to our $A_{i}$ is given by $A_{i}(z)=\tilde{A}_{i}(1-z)$. Note that $A_{i}(-z)=$ $\tilde{A}_{i}(1+z)=\tilde{A}_{i}^{t}(1-z)=A_{i}^{t}(z)$ 
further deduce that $a_{1}$ is a lowering operator for $a_{0}$ and, in fact, $\left\{a_{i}\right\}$ constitutes an irreducible representation of $s l(2, \mathbb{C})$.

To apply this spectral method, the linear equation, (I.3) needs to be transformed to a standard eigenvalue problem with eigenvalue parameter $\zeta$. Actually it suffices to have the coefficient of $\zeta$ be independent of $z$. To accomplish this, take the differential equation associated to (I.3),

$$
\Psi^{\prime}+\left(\frac{1}{2} A_{0}+A_{1} \zeta\right) \Psi=0
$$

and apply a general gauge transformation

$$
\Psi=C(z) \phi
$$

to transform (I.4) to

$$
\phi^{\prime}+\left(C^{-1} C^{\prime}+C^{-1} A_{+} C\right) \phi=0 .
$$

For this to be a standard eigenvalue problem it is necessary that the $C^{-1} A_{1} C$ term in $C^{-1} A_{+} C$ be independent of $z$. If

$$
C^{-1}(z) A_{1}(z) C(z)=A_{1}(0) \quad z \in(-1,1),
$$

then the transformed system becomes

$$
\phi^{\prime}+\left(C^{-1} C^{\prime}+\frac{1}{2} C^{-1} A_{0} C\right) \phi=-A_{1}(0) \zeta \phi
$$

which is an eigenvalue problem. Note that (I.2c) implies that $A_{1}(0)$ is a symmetric matrix.

Next to determine a differential equation to define $C(z)$, observe from the Nahm equation (I.1a) that $A_{1}(z)$ is conjugate to $A_{1}(0)$. But this is precisely what we want $C(z)$ to do. Thus

$$
A_{1}(z)=C(z) A_{1}(0) C^{-1}(z) \quad \text { iff } \quad A_{1}^{\prime}=\left[C^{\prime} C^{-1}, A_{1}\right] .
$$

Comparing with Nahm's equation one deduces

$$
C^{\prime} C^{-1}=\frac{1}{2} A_{0} \text {. }
$$

Equation (I.8) determines $C$ up to multiplication by a constant matrix from the right. This corresponds to the possibility of conjugating Nahm's equations by a constant matrix. Therefore, we require (for consistency with (I.6) in general):

$$
C(0)=I=\text { the identity matrix. }
$$

Then Eq. (I.7) becomes:

$$
\phi^{\prime}+\left(C^{-1} A_{0} C\right) \phi=-A_{1}(0) \zeta \phi .
$$

Another way to characterize the gauge transformation $C(z)$ is to say that it transforms (I.3) into (I.9).

If one defines

$$
\begin{aligned}
Q_{1} & =C^{-1} A_{1} C=A_{1}(0), \\
Q_{0} & =C^{-1} A_{0} C \\
Q_{-1} & =C^{-1} A_{-1} C
\end{aligned}
$$


then Eq. (I.9) becomes

$$
\phi^{\prime}+Q_{0} \phi=-A_{1}(0) \zeta \phi .
$$

This transformation preserves the Lax form of the Nahm equations:

$$
\begin{aligned}
Q_{1}^{\prime} & =0, \\
Q_{0}^{\prime} & =\left[Q_{-1}, Q_{1}\right], \\
Q_{-1}^{\prime} & =\left[Q_{-1}, Q_{0}\right],
\end{aligned}
$$

with $Q_{i} \in \operatorname{sl}(k, \mathbb{C})$.

Observe from Eq. (I.8) that

$$
C^{-1} C^{\prime}=\frac{1}{2} C^{-1} A_{0} C=\frac{1}{2} Q_{0} ;
$$

moreover, since $\left(C^{-1}\right)^{\prime}=-C^{-1} C^{\prime} C^{-1}$, we have

$$
\frac{d}{d z} C^{-1}+\frac{1}{2} Q_{0} C^{-1}=0
$$

with $C^{-1}(0)=I$. So $C^{-1}$ is the normalized fundamental solution of $D+\frac{1}{2} Q_{0}$.

A useful consequence of this reduction is that one can now express the boundary conditions (I.2) for the $A_{i}$ directly in terms of the linear operator $D+\frac{1}{2} Q_{0}$.

Theorem I.1. The following requirements are equivalent to the boundary conditions (I.2) for equation (I.1):

(a) $D+\frac{1}{2} Q_{0}$ (in a neighborhood of the interval $[-1,1]$ ) is an operator with regular singular points (which are simple poles) at $z= \pm 1$ and ordinary points elsewhere. Moreover, the monodromy matrices at these singular points are scalar.

(b) The residue, $q_{0}$, of $Q_{0}$ at $z=1$ together with $q_{1}=A_{1}(0)$ have no common invariant subspaces in $\mathbb{C}^{k}$.

(c) $Q_{0}(-z)=Q_{0}^{t}(z)$.

The proof of this theorem will be deferred to Sect. III, where this characterization will also be related to Donaldson's coordinates on the space of $k$-monopoles.

\section{Solving Nahm's Equations via the Spectral Transform}

The principal ingredients in the explicit solution of the reduced equations (I.11) are the operator ${ }^{5} D+Q_{+}$and the spectral curve of $Q$. The solutions are constructed from meromorphic differentials on this curve and theta functions. This analysis is described in parts A and B. Part C gives a concrete realization of Hitchin's gauge invariant formulation of the boundary conditions in terms of Baker functions on the spectral curve.

A. Asymptotic Analysis of the Normalized Equations. One may take the constant

${ }^{5} Q=Q_{-1} \zeta^{-1}+Q_{0}+Q_{1} \zeta$ and $Q_{+}=Q_{0}+Q_{1} \zeta$ 
symmetric matrix $A_{1}(0)$ of Eq. (I.8ic) to be diagonal:

$$
A_{1}(0)=\text { diagonal }\left(\rho_{1}, \ldots, \rho_{k}\right) \text {. }
$$

Asymptotically, for large $\zeta$, Eq. (I.11) becomes

$$
\phi^{\prime} \cong-\zeta A_{1}(0) \phi \text {. }
$$

The spectral curve associated with Nahm's equation is given by:

$$
S: \operatorname{det}(\eta-A \zeta)=\operatorname{det}(\eta-Q \zeta)=0
$$

which has the form:

$$
p(\eta, \zeta)=\eta^{k}+a_{2}(\zeta) \eta^{k-2}+\cdots+a_{k}(\zeta)
$$

where $\operatorname{deg} a_{i}=2 i$ and the coefficient of $\eta^{k-1}$ is zero since $A, Q \in \operatorname{sl}(k, \mathbb{C})$. By the reality conditions (I.1), the zero set of $p(\eta, \zeta)$ is invariant under the anti-holomorphic involution ${ }^{6}$ :

$$
\tau(\eta, \zeta)=\left(-\bar{\eta} / \bar{\zeta}^{2},-\bar{\zeta}^{-1}\right) .
$$

Hence, the polynomials $a_{i}$ satisfy the "reality" condition:

$$
\zeta^{2 i} \overline{a_{i}(-1 / \bar{\zeta})}=a_{i}(\zeta)
$$

Near $\zeta=\infty$,

$$
\frac{p(\eta, \zeta)}{\zeta^{2 k}} \sim\left(\frac{\eta}{\zeta^{2}}\right)^{k}+\alpha_{2}\left(\frac{\eta}{\zeta^{2}}\right)^{k-2}+\cdots+\alpha_{k} .
$$

The $k$ roots of this polynomial

$$
\rho_{j}=\rho_{j}\left(\alpha_{2}, \ldots, \alpha_{k}\right) \quad j=1, \ldots, k
$$

are the diagonal entries of the matrix $A_{1}(0)$ in Eq. (II.1).

Assume that the $\rho_{i}$ are distinct ${ }^{7} . S$ may be singular; in fact, it may be reducible. However, in this section $S$ will be taken to be non-singular.

Let $\infty_{1}, \ldots, \infty_{k}$ denote the $k$ points of $S$ which lie above $\zeta=\infty$. Let $g$ be the genus of $S$ and let

$$
\delta=\delta_{1}+\cdots+\delta_{g+k-1}
$$

be a positive divisor on $S$ such that

$$
\operatorname{dim}_{\mathbb{C}} \mathscr{L}\left(\delta-\infty_{1}-\cdots-\hat{\infty}_{i}-\cdots-\infty_{k}\right)=1, \quad i=1, \ldots, k,
$$

where $\mathscr{L}(\cdots)$ denotes the space of meromorphic functions on $S$ with poles contained in $\delta$ and zeroes at $\infty_{1}+\cdots+\infty_{i-1}+\infty_{i+1}+\cdots+\infty_{k}$.

\footnotetext{
$6 \bar{\zeta}$ denotes the complex conjugate of $\zeta$.

7 This can be achieved by a linear fractional transformation of $\zeta$ which moves $\infty$. Such a transformation alters the spectral curve and, hence, also Nahm's equations. However, these transformations correspond to rotations in the physical space of the monopoles and so no essential information is lost in our assumption
} 
One can now characterize the eigenfunctions of $D+Q_{+}$as Baker functions. The fundamental existence theorem for the Baker functions is:

Theorem II.1. $[9,10]$ For a given $\delta$ as above, there exist unique functions $\Phi_{j}(z, p)$ for $j=1, \ldots, k(p=(\eta, \zeta)$, a point on $S)$ on $S$ with parameter $z$ in a neighborhood of $z=0$, such that:

(i) $\Phi$ are meromorphic on $S-\left\{\infty_{1}, \ldots, \infty_{k}\right\}$ with poles in $\delta$.

(ii) Near $\infty_{i}, \Phi_{j} \exp \left(\zeta \rho_{i} z\right)$ is holomorphic and equals $\delta_{i j}$ at $\infty_{i}$.

These Baker functions will now be used to construct the reduced Nahm potentials. Let $\phi_{1}, \ldots, \phi_{k}$ be the columns of the fundamental matrix solution, $\Omega$, of Eq. (I.11) normalized so that:

$$
\left.\exp \left(\zeta A_{1}(0) z\right) \Omega\right|_{\zeta=\infty}=\text { identity. }
$$

From the asymptotic structure of (II.1) and the above theorem it follows that $\phi_{l i}$, the $i^{\text {th }}$ component of $\phi_{l}$, is given by:

$$
\phi_{l i}=\Phi_{i}(z, p) \quad \text { for } p \text { near } \quad \infty_{l}=\exp \left(-\zeta \rho_{l} z\right) \sum_{j=0}^{\infty} \alpha_{i l}^{j}(z) \zeta^{-j}, \quad \text { with } \quad \alpha_{i l}^{0}=\delta_{i l} \text {. }
$$

To construct $Q_{0}$ in terms of the coefficients of this expansion, observe that:

$$
\frac{\partial \Phi_{i}}{\partial z}=\exp \left(-\zeta \rho_{l} z\right)\left\{-\delta_{i l} \rho_{l} \zeta+\sum_{j=0}^{\infty}\left(\frac{\partial \alpha_{i l}^{j}}{\partial z}-\alpha_{i l}^{j+1} \rho_{l}\right) \zeta^{-j}\right\}
$$

near $\infty_{k}$. Seek functions

$$
m_{i l}^{j}(z), \quad i, l=1, \ldots, k ; \quad j=0,1 ;
$$

such that:

$$
\frac{\partial \Phi_{i}}{\partial z}-\sum_{v=1}^{k}\left(\zeta m_{i v}^{1}+m_{i v}^{0}\right) \Phi_{v} \sim \exp \left(-\zeta \rho_{l} z\right) \sum_{j=1}^{\infty} h_{i l}^{j}(z) \zeta^{-j}
$$

near $\infty_{l}$. It then follows from a standard argument using the Riemann-Roch theorem (Date [16] page 145) that the last expression is identically zero. From Eq. (II.6) and the condition $\alpha_{i l}^{0}=\delta_{i l}$ (independent of $z$ ),

$$
\begin{aligned}
& m_{i l}^{1}=0 \quad \text { if } \quad i \neq l, \\
& m_{i i}^{1}=-\rho_{i}, \\
& m_{i l}^{0}=\left(\rho_{i}-\rho_{l}\right) \alpha_{i l}^{1}(z),
\end{aligned}
$$

so that:

$$
\frac{\partial \Phi_{i}}{\partial z}-\sum_{v=1}^{k}\left(\rho_{i}-\rho_{v}\right) \alpha_{i v}^{1}(z) \Phi_{v}= \begin{cases}0 & \text { near } \infty_{l}, \quad l \neq i \\ -\zeta \rho_{i} \Phi_{i} & \text { near } \infty_{i}\end{cases}
$$

Therefore:

$$
\left(Q_{0}\right)_{i l}=-\left(\rho_{i}-\rho_{l}\right) \alpha_{i l}^{1}(z)
$$

is the desired construction. Note that the diagonal elements of $Q_{0}$ are zero. 
B. Baker Functions. The functions $\Phi_{j}$ may also be described in terms of meromorphic differentials on $S$. Let $D F K$ denote the differentials of the first kind [17] on $S$. These are 1-forms $\omega$ on $S$ which are everywhere holomorphic. The elements of $D F K$ have the form

$$
\frac{\left(c_{0} \eta^{k-2}+c_{1}(\zeta) \eta^{k-3}+\cdots+c_{k-2}(\zeta)\right) d \zeta}{\partial p / \partial \eta}
$$

where $c_{i}(\zeta)$ is a polynomial of degree $2 i$ and we recall that $p=p(\eta, \zeta)$, Eq. (II.2a), defines the curve $S$. One may relate this expression to $\eta$ by the identity

$$
\frac{d \zeta}{\partial p / \partial \eta}=-\frac{d \eta}{d p / \partial \zeta}
$$

The genus of $S$ is equal to the dimension of DFK. From Eq. (II.11),

$$
g=(k-1)^{2} \text {. }
$$

We will now list and describe the basic ingredients involved in constructing the $\Phi_{j}$ 's.

(i) Differentials of the Second Kind:

It follows from Eqs. (II.2c, d) that the rational function $\eta / \zeta$ has the asymptotic behavior

$$
\eta / \zeta \sim \rho_{j} \zeta \quad \text { near } \infty_{j}
$$

and a similar analysis shows that

$$
\eta / \zeta \sim \sigma_{j} \zeta^{-1} \text { near } 0_{j}
$$

where $0_{j}, j=1, \ldots, k$, are the $k$ points of $S$ which lie over $\zeta=0$ and $\sigma_{j}=-\bar{\rho}_{j}$. Elsewhere $\eta / \zeta$ is analytic. Therefore, the exact differential $d(\eta / \zeta)$ has $k$ double poles, without residues, at $\infty_{1}, \ldots, \infty_{k}$, and has $k$ double poles, without residues, at $0_{1}, \ldots, 0_{k}$; i.e. in terms of local coordinate $t$ :

$$
\begin{aligned}
d\left(\frac{\eta}{\zeta}\right) & \sim\left(-\frac{\rho_{j}}{t^{2}}+O(1)\right) d t \quad \text { near } \infty_{j} \\
& \sim\left(-\frac{\sigma_{j}}{t^{2}}+O(1)\right) d t \quad \text { near } 0_{j}
\end{aligned}
$$

Motivated by this, we define a differential $\gamma_{\infty}$ which satisfies:

$$
\begin{gathered}
\gamma_{\infty} \sim\left(\frac{\rho_{j}}{t^{2}}+O(1)\right) d t \quad \text { near } \infty_{j} ; \\
\oint_{a_{\mathrm{l}}} \gamma_{\infty}=0,
\end{gathered}
$$$$
\gamma_{\infty} \text { holomorphic away from } \infty \text {. }
$$

Because (II.13b) imposes $g$ conditions on these differentials of the second kind, $\gamma_{\infty}$ exists and is unique. However, unlike $d(\eta / \zeta), \gamma_{\infty}$ is not exact. $\gamma_{\infty}$ encodes the asymptotic behavior of $d \log \Phi_{j}$ for large $\zeta$. 
(ii) The Abel Map:

is defined by

$$
W: S \rightarrow \mathbb{C}^{g}
$$

$$
W(p)=\int_{p_{0}}^{p} \vec{\omega}
$$

where $p_{0}$ is a fixed base point, $p$ is an arbitrary point of $S$ and $\vec{\omega}=\left(\omega_{1}, \ldots, \omega_{g}\right)^{t}$, where $\left\{\omega_{i}\right\}$ is a basis for $D F K$. This is defined, up to periods, whether $S$ is singular or not. When $S$ is non-singular, it is natural to choose a normalized basis for $\omega$ :

$$
\oint_{a_{\imath}} \omega_{j}=\delta_{i j}
$$

The numbers

$$
B_{i j}=\oint_{b_{\imath}} \omega_{j}
$$

are the entries of the Riemann matrix $B . B$ is symmetric with positive definite imaginary part. It is natural to regard $W$ as a map from $S$ to $\mathbb{C}^{g} / \Lambda$, where $\Lambda$ is the lattice in $\mathbb{C}^{g}$ spanned by the $2 g$ columns of $(I \mid B) . \mathbb{C}^{g} / \Lambda$ is a $g$-dimensional torus called the Jacobian variety of $S$ or $\operatorname{Jac}(S)$. The Abel map $W$ relates the geometry of $S$ to that of $\operatorname{Jac}(S)$.

(iii) Riemann's Theta Function:

This is an entire analytic function $\Theta(\vec{z})$ on $C^{g}$ :

$$
\Theta(\vec{z})=\sum_{\vec{m} \in \mathbb{Z}^{g}} \exp [\pi i\{\langle B \vec{m}, \vec{m}\rangle+2\langle\vec{z}, \vec{m}\rangle\}] .
$$

This function is automorphic with respect to the periods in $\Lambda$ :

$$
\begin{aligned}
& \Theta\left(\vec{z}+A_{i}\right)=\Theta(\vec{z}), \\
& \Theta\left(\vec{z}+n_{1} B_{1}+\cdots+n_{g} B_{g}\right)=\exp \{-\pi i(\langle B \vec{n}, \vec{n}\rangle+2\langle\vec{z}, \vec{n}\rangle)\} \Theta(\vec{z}),
\end{aligned}
$$

where $\vec{n}=\left(n_{1}, \ldots, n_{g}\right)^{t}$ and the vectors $A_{i}, B_{i}$ are the integrals of $\vec{\omega}$ along the $a_{i}$ and $b_{i}$ loops respectively; i.e., $B_{i}$ are the columns of the Riemann matrix $B$. Note that $A_{i}$ are just the unit vectors $\vec{e}_{i}$ if $\omega$ is normalized.

A fundamental result which relates $\Theta$ to the function theory of $S$ is

Riemann's Vanishing Theorem. ${ }^{8}$ There is a vector $K \in \mathbb{C}^{g}$, such that for all $\vec{z} \in \mathbb{C}^{g}$, $f(\vec{z})=\Theta(\vec{z}+W(p))$ either vanishes identically, or has $g$ zeros $q_{1}, \ldots, q_{g}$ such that

$$
W\left(q_{1}\right)+\cdots+W\left(q_{g}\right) \equiv-\vec{z}+K \bmod \Lambda .
$$

(iv) Normalization Factors:

We now introduce several functions and constants which will be used in our construction to insure that the normalization conditions in (ii) of Theorem II.1 are satisfied. Let $\delta$ be an effective divisor on $S$ of degree $g+k-1=$

\footnotetext{
${ }^{8}$ see Siegel [18] for an explicit expression of $K$
} 
$k(k-1)$ which satisfies (II.3). Then there are unique rational functions $g_{i}$ in $\mathscr{L}\left(\delta-\infty_{1}-\cdots-\hat{\infty}_{i}-\cdots-\infty_{k}\right)$ (this is the space of rational functions on $S$ with poles in $\delta$ and having zeros at $\left.\infty_{1}+\cdots+\hat{\infty}_{i}+\cdots+\infty_{k}\right)$ such that $g_{i}\left(\infty_{i}\right)=1$. The divisor of $g_{i}$,

$$
\left(g_{i}\right)=\Delta_{i}+\infty_{1}+\cdots+\hat{\infty}_{i}+\cdots+\infty_{k}-\delta,
$$

where $\Delta_{i}$ is the unique divisor of degree $g$ consisting of the residual zeros of $g_{i}$.

Next we define the normalization constants:

$$
v_{j}=\lim _{p \rightarrow \infty}\left[\left(\int_{p_{0}}^{p} \gamma_{\infty}\right)+\eta / \zeta\right]
$$

where $\zeta$ is, naturally, regarded as a function on $S$. Because of the asymptotics (II.13a), the $v_{j}$ are finite numbers. Note that $v_{j}$ depends on the basepoint $p_{0}$, just as Riemann's constant $K$ depends on the basepoint of the Abel map.

Using the above ingredients one can now present an explicit formula for $\Phi_{j}$ :

$$
\begin{aligned}
\Phi_{j}(z, p)= & g_{j}(p) \exp \left\{\left(z \int_{p_{0}}^{p} \gamma_{\infty}\right)-\left(v_{j} z\right)\right\} \\
& \cdot \frac{\Theta\left(W(p)-\vec{\kappa} z-W\left(\Delta_{j}\right)-K\right) \Theta\left(W\left(\infty_{j}\right)-W\left(\Delta_{j}\right)-K\right)}{\Theta\left(W(p)-W\left(\Delta_{j}\right)-K\right) \Theta\left(W\left(\infty_{j}\right)-\vec{\kappa} z-W\left(\Delta_{j}\right)-K\right)},
\end{aligned}
$$

where $W\left(p_{1}+\cdots+p_{n}\right)=W\left(p_{1}\right)+\cdots+W\left(p_{n}\right)$. The "frequency" vector $\vec{\kappa}$ is uniquely determined by the condition that $\Phi_{j}$ should be a function on $S$ (as will be shown later in Eq. (II.34b), $\vec{\kappa}$ is a vector with half-integer components). This amounts to requiring that $\Phi_{j}$ remain unchanged when we transport $p$ around any cycle in the homology basis for $S$. By the normalization condition (II.13b) and the periodicity (II.16a) of the $\Theta$-functions, this is already satisfied for the $a$-cycles. However, if we transport $p$ around a $b$-cycle, then $\Phi_{j}(z, p)$ gets multiplied by the factor

$$
\left(\exp \oint_{b_{j}} z \gamma_{\infty}\right) \times\left(\exp \left(-(2 \pi i) \kappa_{j} z\right)\right)
$$

Since this factor must be 1 ,

$$
\kappa_{j}=\frac{1}{2 \pi i} \oint_{b_{j}} \gamma_{\infty} \quad j=1, \ldots, g .
$$

We will now check that these functions satisfy the conditions of Theorem II.1. Since the theta function is analytic, the theta ratio in (II.19) must be meromorphic on $S$. The function $g_{i}$ is meromorphic by construction. On $S-\left\{\infty_{1}, \ldots, \infty_{k}\right\}$ the leading exponential factor has no singularities. Hence, $\Phi_{j}$ is meromorphic in $S-\left\{\infty_{1}, \ldots, \infty_{k}\right\}$. The poles of $g_{i}$ all lie in $\delta$. However, the zeros of the theta function in the denominator of (II.19) introduce poles. Now, by Riemann's vanishing theorem, $\Theta\left(W(p)-W\left(\Delta_{j}\right)-K\right)$ is zero precisely at the $g$ points in $\Delta_{j}$. But by (II.17) $g_{j}$ has zeros at each of these points which cancel the potential singularity. Hence, $\Phi_{j}$ has its poles inside $\delta$. Next, consider $\exp \left(z \rho_{l} \zeta\right) \Phi_{j}(z, p)$. This is holomorphic near $\infty_{l}$ by the asymptotics (II.13a). Moreover, it goes to zero as $p$ goes to $\infty_{l}$ if $l \neq j$, since $g_{j}\left(\infty_{l}\right)=\delta_{j l}$. Finally, if $j=l$, then as $p$ goes to $\infty_{l}, g_{j}\left(=g_{l}\right)$ goes to 1 , and the 
exponential factor also goes to 1 . Therefore, all the conditions in Theorem II.1 are satisfied by these $\Phi_{j}$.

Finally we can construct $Q_{0}$. Recall that from (II.10)

$$
\left(Q_{0}\right)_{i j}=-\left(\rho_{i}-\rho_{j}\right) \alpha_{i j}^{1}(z) \text {. }
$$

Therefore, using (II.5), for $i \neq j$,

$$
\begin{aligned}
\alpha_{i j}^{1}= & \lim _{p \rightarrow \infty_{j}} \zeta \exp \left(z \rho_{j} \zeta\right) \Phi_{i}=c_{i j} \exp \left\{\left(v_{j}-v_{i}\right) z\right\} \\
& \cdot \frac{\Theta\left(W\left(\infty_{j}\right)-\vec{\kappa} z-W\left(\Delta_{i}\right)-K\right) \Theta\left(W\left(\infty_{i}\right)-W\left(\Delta_{i}\right)-K\right)}{\Theta\left(W\left(\infty_{j}\right)-W\left(\Delta_{i}\right)-K\right) \Theta\left(W\left(\infty_{i}\right)-\vec{\kappa} z-W\left(\Delta_{i}\right)-K\right)},
\end{aligned}
$$

where $c_{i j}=\lim _{p \rightarrow \infty_{j}} \zeta g_{i}(p)$, is a finite constant since $g_{i}$ vanishes at $\infty_{j}$ and we have used:

$$
\lim _{p \rightarrow \infty_{j}}\left(\rho_{j} \zeta+\int_{p_{0}}^{p} \gamma_{\infty}\right)=v_{j} .
$$

Note that due to the factor $\left(\rho_{j}-\rho_{i}\right), \alpha_{i i}^{1}$ does not contribute to $Q_{0}$.

C. Gauge Invariant Boundary Conditions. Hitchin showed that the monopole conditions encoded in Nahm's equations (I.1 and I.2) could be formulated purely in terms of the spectral curve $S$ and a one-parameter family of line bundles, $L^{s}$, on that curve. $L^{s}$ is defined by the transition function

$$
\phi_{12}=\exp (-s \eta / \zeta) \text { in } U_{1} \cap U_{2},
$$

where

$$
\begin{aligned}
& U_{1}=S \cap\{(\eta, \zeta) \mid \zeta \neq \infty\}, \\
& U_{2}=S \cap\{(\eta, \zeta) \mid \zeta \neq 0\} .
\end{aligned}
$$

Let $L$ denote $L^{1}$ and set $z=s-1$. Also, take $\delta$ to be the divisor associated to the line bundle $L(k-1)$; condition (II.3) then becomes equivalent to

$$
\operatorname{dim}_{\mathbb{C}} \mathscr{L}\left(L(k-2) \otimes \mathcal{O}\left(\infty_{i}\right)\right)=1, \quad i=1, \ldots, k,
$$

where $\mathcal{O}\left(\infty_{i}\right)$ is the line bundle associated to the divisor $\infty_{i}$ and $\mathscr{L}(\cdots)$ denotes "the space of holomorphic sections of." If (II.22) is satisfied then $\operatorname{dim}_{\mathbb{C}} \mathscr{L}\left(L^{z}(k-1)\right)=k$ for $z$ in some neighborhood of $z=0$ and the Baker functions $\Phi_{j}(z, p)$ correspond to a basis of the space of sections $\mathscr{L}\left(L^{z}(k-1)\right)$ under the association of $\Phi_{j}$ to the section which is

$$
\text { and } \begin{array}{lll}
1 & \text { on } & U_{2} \\
\Phi_{j}(z, p) & \text { on } & U_{1}-\{\delta\}
\end{array}
$$

Hitchin's boundary conditions, which will be further explained in the subsequent discussion, are

(i) $L(k-1)$ is real with respect to the anti-holomorphic structure induced by (II.2b).

(ii) $L^{2}$ is trivial on $S$.

(iii) $\mathscr{L}\left(L^{s}(k-2)\right)=0$ for $s \in(0,2)$. 
These conditions are the equivalent of (I.2) (see Hitchin [7]) under the implicit assumptions that $S$ is non-singular and $L$ satisfies (II.22). In what follows we will point out where these assumptions enter. Let us examine (II.23) one condition at a time.

(i) A basis for $\mathscr{L}(L(k-1))$ is given by

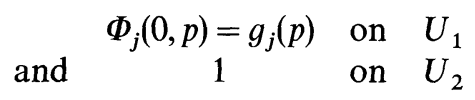

for $j=1, \ldots, k$.

The induced anti-holomorphic involution, $\sigma$, on these sections is given by

$$
\begin{array}{ccc}
1 & \text { on } & U_{1} \\
g_{j}(\tau p) & \text { on } & U_{2}-\{\tau \delta\} .
\end{array}
$$

Clearly, $\sigma^{2}$ is the identity on these sections and therefore $L(k-1)$ is real (see [19]).

(ii) To say $L^{2}$ is trivial means that one can find functions 9

$$
\begin{gathered}
f_{1} \text { holomorphic on } U_{1}, \\
f_{2} \text { holomorphic on } U_{2}, \\
\text { such that } f_{2}=\phi_{12} f_{1}=\exp (-2 \eta / \zeta) f_{1} \text { on } U_{1} \cap U_{2} .
\end{gathered}
$$

Taking the logarithmic derivative of (II.24c) gives

$$
d \log f_{2}=d(-2 \eta / \zeta)+d \log f_{1} .
$$

If $f_{2}$ is not to have an essential singularity on $U_{2}$, then it follows from (II.24c, d) that $d \log \left(f_{1}\right)$ must cancel the poles of $d(-2 \eta / \zeta)$ at $\infty_{j}$. Hence, using (II.12a),

$$
d \log f_{1} \sim\left(2 \frac{\rho_{j}}{t^{2}}+O(1)\right) d t \quad \text { near } \infty_{j} .
$$

If $f_{1}$ is to be a function on $U_{1}$, one must have

$$
\exp \oint_{\lambda} d \log f_{1}=1 \text { for all } \lambda \in \Lambda,
$$

where $\Lambda$ is the group of all finite integral linear combinations generated by the homology basis $a_{1} \ldots a_{g} ; b_{1} \ldots b_{g}$ on $S$. (Even though $U_{1}$ is $S$ with $k$ points deleted, the homology group of one cycles on $S$ does not acquire any linear relations when restricted to $U_{1}$.) Normalize $d \log f_{1}$ so that

$$
\oint_{a_{i}} d \log f_{1}=0 \text {. }
$$

As discussed in (II.13), a differential, $d \log f_{1}$ which satisfies (II.27) and is holomorphic in $U_{1}$ must be unique. In fact,

$$
d \log f_{1}=-2 \gamma_{\infty} .
$$

\footnotetext{
9 For the case $k=2$, a similar construction, also based on meromorphic differentials, may be found in Hurtubise [20]
} 
Now to also satisfy the full condition (II.26) it is necessary and sufficient that

$$
\oint_{b_{j}} \gamma_{\infty}=\pi i m_{j} \text { for some } m_{j} \in \mathbb{Z} \text {. }
$$

This imposes $g$ conditions on the space of all Nahm spectral curves. Equation (II.29) is equivalent to the condition (II.23ii); however, since these are transcendental conditions, one does not know that this condition can be satisfied for a non-singular curve when $k>2$.

It will be convenient later to have defined another differential related to $d \log f_{2}$ :

$$
\gamma_{0}=d(-\eta / \zeta)-\gamma_{\infty} .
$$

A special case of this condition, for $s=1$, is $\mathscr{L}(L(k-2))=0$ which is equivalent to (II.22). In classical language the full condition (iii) states that the divisor associated to $L^{s}(k-2)$ is non-special for $s \in(0,2)$ and hence the Baker functions have no singularities in this range. At the present time this condition must be assumed.

Using (II.23) we can streamline the functional representation of Baker's function and hence of the potential $Q_{0}$. Firstly we have

$$
v_{j}=-\int_{p_{0}}^{\infty} \gamma_{0}
$$

which follows from inserting (II.30) into the definition of $v_{j}$ (II.18).

Next, combining (II.22) with (II.17), there is an isomorphism of line bundles

$$
\mathcal{O}\left(\Delta_{i}\right) \equiv L(k-2) \otimes \mathcal{O}\left(\infty_{i}\right)
$$

which means that the associated divisors are linearly equivalent and, by Abel's theorem, they have the same values under the Abel map. Thus

$$
W\left(\Delta_{i}\right)=\vec{\kappa}+W\left((k-2)\left(\infty_{1}+\cdots+\infty_{k}\right)\right)+W\left(\infty_{i}\right) .
$$

Here one uses the identity between the Abel image of the divisor of $L$ and the characteristics of $L(=\vec{\kappa})$. Taking $(k-2)\left(\infty_{1}+\cdots+\infty_{k}\right)$ to be the basepoints of the Abel map on divisors of degree $g-1(=k(k-2))$, one may write

$$
\vec{\kappa}=W\left(\Delta_{j}\right)-W\left(\infty_{i}\right) \text { for all } i
$$

Since

$$
\kappa_{j}=\frac{m_{j}}{2}
$$

for some integer $m_{j}$ (see Eqs. (II.29) and (II.20)),

$$
\vec{\kappa}=\frac{m_{1}}{2} A_{1}+\cdots+\frac{m_{g}}{2} A_{g},
$$

where $A_{i}$ are the $a_{i}$-periods of the Abel map.

So, finally, adopting characteristic notation (see Fay [21]) with $\vec{m}=\left(m_{1}, \ldots, m_{g}\right)^{t}$, 


$$
\begin{aligned}
\Phi_{j}(z, p)= & g_{j}(p) \exp \left\{z\left(\int_{p_{0}}^{p} \gamma_{\infty}+\int_{p_{0}}^{\infty_{j}} \gamma_{0}\right)\right\} \\
& \cdot \frac{\Theta\left[\begin{array}{c}
\vec{m} \\
0
\end{array}\right]\left(W(p)-W\left(\infty_{j}\right)-\vec{\kappa} z-K\right) \Theta\left[\begin{array}{c}
\vec{m} \\
0
\end{array}\right](K)}{\Theta\left[\begin{array}{c}
\vec{m} \\
0
\end{array}\right]\left(W(p)-W\left(\infty_{j}\right)-K\right) \Theta\left[\begin{array}{c}
\vec{m} \\
0
\end{array}\right](\vec{\kappa} z+K)}
\end{aligned}
$$

where we used the fact that $\left[\begin{array}{c}\vec{m} \\ 0\end{array}\right]$ is an even characteristic (this means that these $\Theta$-functions are "even," $\Theta\left[\begin{array}{c}\vec{m} \\ 0\end{array}\right](-\vec{x})=\Theta\left[\begin{array}{c}\vec{m} \\ 0\end{array}\right](\vec{x})$; see Fay [21]) to remove some minus signs.

Note that when $z= \pm 1$,

$$
\begin{aligned}
\Theta\left[\begin{array}{c}
\vec{m} \\
0
\end{array}\right](\vec{\kappa}+K) & =\text { non-vanishing factor } \times \Theta(K) \text { by (II.34a) } \\
& =0 .
\end{aligned}
$$

So $\Phi_{j}(z, p)$ has a singularity at $z= \pm 1$.

The symmetry condition (I.2c) becomes a normalization for Baker functions. Precisely, if one rewrites (II.21) using the characteristic notation of (II.35), then, because $\Theta\left[\begin{array}{c}\vec{m} \\ 0\end{array}\right](\vec{x})$ is even, the symmetry condition reduces to the requirement that

$$
c_{i j}=-c_{j i} \text {. }
$$

We end this section with another useful characterization of the "periodic" quantization conditions in terms of the normalized holomorphic differentials. First, some notation. Considering the normalized differentials $\omega_{i}$, near $\infty_{l}$, we have $\vec{\omega}=\vec{f}^{(l)}(t) d t$, where $\vec{f}^{(l)}(t) d t$ is analytic near $t=0$. Let $\vec{C}^{(l)}=\left(c_{1}^{(l)}, \ldots, c_{g}^{(l)}\right)^{t}=\vec{f}^{(l)}(0)$.

\section{Theorem II.2.}

(i) $L^{2}$ is trivial on $S$ iff

$$
\sum_{j=1}^{k} 2 \rho_{j} \vec{C}^{(j)} \in \mathbb{Z}^{g}
$$

where $\rho_{j}$ is given by (II.1) or (II.12a).

(ii) $H^{0}\left(S, L^{s}(k-2)\right)=0 \Rightarrow H^{0}\left(S, L^{s}\right)=0$

$$
\Leftrightarrow \sum_{j=1}^{k} 2 \rho_{j} \vec{C}^{(j)} \text { is a generator of } \mathbb{Z}^{g} \text {. }
$$

Proof. By Riemann's bilinear identity for differentials of the first and second kind (see e.g. Springer [17], p. 260):

$$
\sum_{j=1}^{g}\left(\omega_{l}\left(a_{j}\right) \gamma_{\infty}\left(b_{j}\right)-\omega_{l}\left(b_{j}\right) \gamma_{\infty}\left(a_{j}\right)\right)=2 \pi i \sum_{l=1}^{k} 2 \rho_{l} c_{i}^{(l)}
$$


Using $\gamma_{\infty}\left(a_{j}\right)=0$, the normalization $\omega_{i}\left(a_{j}\right)=\delta_{i j}$ and the quantization condition (II.29) we have:

$$
\sum_{l=1}^{k} 2 \rho_{l} c_{i}^{(l)}=n_{i}
$$

Conversely, from the quantization condition one can construct a trivializing section. This proves part (i) of the theorem.

By the reasoning used before, $H^{0}\left(S, L^{s}\right)=0$ is equivalent to

$$
s\left(\sum_{l=1}^{k} 2 \rho_{l} C^{(l)}\right) \notin \mathbb{Z}^{g}, \quad s \in(0,2) \text {. }
$$

Since,

$$
2\left(\sum_{l=1}^{k} 2 \rho_{l} C^{(l)}\right) \in \mathbb{Z}^{g},
$$

by part (i), it follows that this must be a generator.

\section{Monodromy of the Linear Operator}

In part $\mathrm{A}$ of this section we prove Theorem I.1. This involves comparing the linear operators $D-\frac{1}{2} A_{0}$ and $D+\frac{1}{2} Q_{0}$, and in particular their monodromy at the regular singular points $z= \pm 1$.

In part B the singular solutions of the linear operators (I.3) and (I.11), evaluated at $\zeta=0$ (these are $D+\frac{1}{2} A_{0}$ and $D+Q_{0}$ respectively) near $z=1$ provide data for constructing an invariant of the monopole. This is Donaldson's rational map $f: \mathbb{C} \mathbb{P}^{1} \rightarrow \mathbb{C} \mathbb{P}^{1}$ of degree $k$ such that $f(\infty)=0$. Donaldson [4] shows that the space of such maps is a circle bundle over the moduli space $M_{k}$ of $k$-monopoles. Our constructions show how to define this map in terms of the Baker functions of $D+Q_{0}$.

\section{A. Proof of Theorem I.1.}

Theorem I.1. The following requirements are equivalent to the boundary conditions (I.2) for Eq. (I.1).

(a) $D+\frac{1}{2} Q_{0}$ (in a neighborhood of the interval $[-1,1]$ ) is an operator with regular singular points (which are simple poles) at $z= \pm 1$ and ordinary points elsewhere. Moreover, the monodromy matrices at these singular points are scalar.

(b) The residue, $q_{0}$, of $Q_{0}$ at $z=1$ together with $q_{1}=A_{1}(0)$ have no common invariant subsapces in $\mathbb{C}^{k}$.

(c) $Q_{0}(-z)=Q_{0}^{t}(z)$.

The equivalence of (c) in the theorem to (I.2c) is based on:

Lemma III.1. If $A_{0}(-z)=A_{0}^{t}(z)$, then $C(-z)=\left(C^{t}(z)\right)^{-1}$.

One easily checks that, if $A_{0}(-z)=A_{0}^{t}(z)$, then both sides of this equation satisfy

$$
X^{\prime}+\frac{1}{2} A_{0}(-z) X=0 \text {. }
$$


Since $C(0)=I$, both sides satisfy the initial condition $X(0)=I$.

Since $Q_{0}(z)=C^{-1}(z) A_{0}(z) C(z)$, we have

$$
Q_{0}=C^{-1}(-z) A_{0}(-z) C(-z)
$$

On the other hand

$$
\begin{aligned}
Q_{0}^{t}(z) & =C^{t}(z) A_{0}^{t}(z)\left(C^{t}(z)\right)^{-1} & & \\
& =C^{t}(z) A_{0}(-z)\left(C^{t}(z)\right)^{-1} & & \text { by (I.2c) } \\
& =(C(-z))^{-1} A_{0}(-z) C^{t}(-z) & & \text { by the lemma. }
\end{aligned}
$$

Hence (I.2c) implies (c) in the theorem.

An analogous argument shows that the conclusion of the lemma also follows from (c) and, hence, so does I.2(c) for $A_{0}$, essentially by interchanging $A_{0}$ and $Q_{0}$ in the preceding paragraph. A similar argument also proves I.2(c) for $A_{1}$ once we observe that $A_{1}=A_{1}(0)$ is symmetric and independent of $z$.

The discussion of condition (a) will be based on a trivial lemma concerning the monodromy matrices of (I.8) and (I.13). Let $C(z)$ be the fundamental solution of $\left(D-\frac{1}{2} A_{0}\right) C=0$ normalized so that $C(0)=I$. If $A_{0}(z)$ is analytic on $(-1,1)$, so is $C(z)$ which, in addition is invertible there. Hence $Q_{0}=C^{-1} A_{0} C$ is also analytic on $(-1,1)$. A similar argument based on (I.13) proves the converse.

At $z= \pm 1$ we assume (I.2a,b); viz., that $A_{0}(z)$ is meromorphic with simple poles, and further that the residues $a_{0}, a_{1}$ have no non-trivial invariant subspaces. Let $\gamma$ be a circuit around $z=1$ (the argument for $z=-1$ is similar). $C(\gamma z)$ denotes the transport of the fundamental solution around this circuit; $C(\gamma z)=\Gamma C(z)$ and $\Gamma$, a $z$-independent matrix, is called the monodromy matrix of $\gamma$. One can now develop the lemma mentioned above.

Lemma III.2. The monodromy matrices for the fundamental solution, $C(z)$, of $\left(D-\frac{1}{2} A_{0}\right) C=0$ are scalar matrices.

Since $A_{0}=2 C^{\prime} C^{-1}$ and $A_{1}=C A_{1}(0) C^{-1}$, the monodromy of $A_{i}$ is induced from that of $C$ :

$$
A_{i}(\gamma z)=\Gamma A_{i}(z) \Gamma^{-1} .
$$

In particular, $a_{i}=\Gamma a_{i} \Gamma^{-1}$. But by Remark I.1 $\{a\}$ is an irreducible representation of $s l(2, \mathbb{C})$; hence, by Schur's lemma, $\Gamma$ must be scalar.

To apply this lemma, observe that since $A_{0}(z)$ has a simple pole at $z=1, z=1$ is a regular singular point of $D-\frac{1}{2} A_{0}$. By Remark I.1 this operator has a fundamental set of basis elements for its kernel which near $z=1$ start like $(z-1)^{(k-2 j+1) / 2}, j=1, \ldots, k$. Because these exponents differ by integers, the full series expansion of these solutions at $z=1$ will have resonances. However, by the lemma the monodromy matrix is diagonal and hence the series may be continued without the introduction of logarithmic terms. Therefore, the fundamental solution of (I.8) has algebraic exponents (in fact, they are half-integer) near $z=1$.

From this it follows that the $Q_{i}$ have Puiseux expansions in $(z-1)$; but, granted this, from Eqs. (I.12) one sees that the $Q_{i}$ go like

$$
Q_{0}=\frac{q_{0}}{z-1}+\cdots, \quad Q_{-1}=\frac{q_{-1}}{(z-1)^{2}}+\cdots,
$$

so that $D+\frac{1}{2} Q_{0}$ has a regular singular point (simple pole) at $z=1$. By (I.13) the 
fundamental solution of this operator is $C^{-1}(z)$ which, by Lemma III.2, has scalar monodromy.

The proof of the converse, that the conditions of Theorem I.1 imply (I.2a), is entirely similar to the previous argument.

Finally, condition (b) of Theorem I.1 is equivalent to (I.2b). Suppose that there is a $z$-independent $\Gamma$ such that

$$
\Gamma^{-1} q_{i} \Gamma=q_{i} \text { for } i=0,1 .
$$

Then, by (I.12),

$$
\Gamma^{-1} Q_{i}(z) \Gamma=Q_{i}(z) \text { for } i=0,1 .
$$

Conjugate (I.13) by $\Gamma$,

$$
\left(\Gamma C^{-1} \Gamma^{-1}\right)^{\prime}=-\frac{1}{2} \Gamma Q_{0} \Gamma^{-1}\left(\Gamma C^{-1} \Gamma^{-1}\right)=-\frac{1}{2} Q_{0}\left(\Gamma C^{-1} \Gamma^{-1}\right) .
$$

Since $\Gamma C^{-1}(0) \Gamma^{-1}=I$,

$$
C^{-1}=\Gamma C^{-1} \Gamma^{-1}
$$

But

$$
\begin{aligned}
A_{i}=C Q_{i} C^{-1} & =\Gamma C \Gamma^{-1} Q_{i} \Gamma C^{-1} \Gamma^{-1} & & \text { by (III.4) } \\
& =\Gamma C Q_{i} C^{-1} \Gamma^{-1} & & \text { by (III.3) } \\
& =\Gamma A_{i} \Gamma^{-1} & &
\end{aligned}
$$

This implies that $\Gamma a_{i} \Gamma^{-1}=a_{i}$ for $i=0,1$; hence, $\Gamma$ must be a scalar by the irreducibility of $\left\{a_{0}, a_{1}\right\}$. The converse is similar.

B. Donaldson's Rational Map. Our version of Donaldson's construction is based on a consequence of Theorem I.1:

Corollary II.3. $-(k-1)$ is an eigenvalue of the residue $q_{0}$.

Proof. In an eigenbasis, $a_{0}$ may be taken to have the form (see Remark I.1):

$$
a_{0}=\left(\begin{array}{ccc}
k-1 & & 0 \\
0 & & -(k-1)
\end{array}\right) \text {. }
$$

For $\zeta=0,(\mathrm{I} .4)$ is

$$
\left(D+\frac{1}{2} A_{0}\right) \Psi=0
$$

Expanded near $z=1$ this becomes

$$
(z-1) \Psi^{\prime}=-\frac{1}{2}\left[a_{0}+(z-1) a_{1}+\cdots\right] \Psi .
$$

Related to the eigenbasis of $a_{0}$ this has fundamental solutions

$$
\Psi_{j}=(z-1)^{-((k-2 j+1) / 2)}\left\{e_{j}+\vec{\xi}_{j 1}(z-1)+\vec{\xi}_{j 2}(z-1)^{2}+\cdots\right\} .
$$

Applying the gauge transformation, $C(z)$, to this (recall that $\left.\left(D-\frac{1}{2} A_{0}\right) C=0\right)$, we get

$$
\Psi_{j}=C \vec{v}_{j},
$$


which relates $\vec{\Psi}_{j}$ to a function $\vec{v}_{j}(z)$ which solves

$$
\left(D+Q_{0}\right) \vec{v}_{j}=0 \text {. }
$$

From now on we restrict attention to $\vec{v}_{1}$. Near $z=1$,

$$
\begin{aligned}
\vec{v}_{1} & =C^{-1} \Psi_{1} \\
& =(z-1)^{-((k-1) / 2)} F(z) \Psi_{1}, \quad \text { with } F(z) \text { analytic near } z=1 \\
& =(z-1)^{-(k-1)} F(z)\left(e_{1}+O(z-1)\right) .
\end{aligned}
$$

The second line holds because $\left(C^{-1}(z)\right)^{t}$ solves

$$
X^{\prime}+\frac{1}{2} A_{0}^{t}(z) X=0
$$

and, since $a_{0}$ is diagonal, this has the same asymptotic behavior near $z=1$ as (III.6). Furthermore, by the form of (III.6),

$$
F(z)=\left(\begin{array}{cccc}
1 & 0 & \cdots & 0 \\
0 & & & \\
\vdots & & & \\
0 & & &
\end{array}\right)+O(z-1)
$$

Hence,

$$
\vec{v}_{1}(z)=(z-1)^{-(k-1)} e_{1}+O(z-1) .
$$

Evaluating (III.9) on (III.10),

$$
D\left((z-1)^{-(k-1)} e_{1}+\cdots\right)-\left(\frac{q_{0}}{z-1}+\cdots\right)\left((z-1)^{-(k-1)} e_{1}+\cdots\right)=0
$$

whose leading terms give the relation

$$
q_{0} e_{1}=-(k-1) e_{1} .
$$

Now recall Donaldson's construction. Referring to the previous proof one defines the vector

$$
w=\Psi_{1}(z=0) \text {. }
$$

Also, set $B=A_{1}(0)=Q_{1}$. Then Donaldson's map is the rational function

$$
f(\lambda)=w^{t}(\lambda I-B)^{-1} w .
$$

However, since $\Psi_{1}(z)=C(z) \vec{v}_{1}(z)$ and $C(0)=I$,

$$
f(\lambda)=\left(\vec{v}_{1}(0)\right)^{t}\left(\lambda I-Q_{0}\right)^{-1} \vec{v}_{1}(0)
$$

where $Q_{0}=$ diagonal $\left(\rho_{1}, \ldots, \rho_{k}\right)$ is determined by the curve equation (II.2). Since $\vec{v}_{1}$ solves (III.9) it is expressible in terms of the Baker functions $\Phi_{j} . w=\vec{v}_{1}(0)$ is a linear combination of Baker vectors at $z=0$ and $\zeta=0$. Hence

$$
w=\sum_{j=1}^{k} c_{j} \vec{g}_{j}
$$


where $\vec{g}_{j}=\left(g_{j}\left(0_{1}\right), \ldots, g_{j}\left(0_{k}\right)\right)^{t}$, the $g_{j}(p)$ are given by (II.17) and the $c_{j}$ are determined implicitly by (III.10).

\section{Elliptic Solutions of Nahm Equations}

This section begins by describing a class of solutions of Nahm's equations, that can be written in terms of Jacobian elliptic functions. ${ }^{10}$ These solutions generalize those of Prasad and Rossi [23]; Nahm [2]; Brown et al. [24]. It is conjectured that they correspond to equally spaced monopoles with centers located along the $x_{2}$-axis (see [24] for $k=2$ ).

A. The Elliptic Solutions. It is well known that, up to equivalences, the Lie algebra $s u(2)$ has a unique $k$-dimensional irreducible representation. Choosing a basis of this representation in $k \times k$ hermitian matrices $J_{a}=\left(J_{a}\right)^{*}, a=1,2,3$, let us also define $J_{ \pm}=J_{1} \pm i J_{2}$, so that $J_{+}=\left(J_{-}\right)^{*}$. They satisfy the commutation relations:

$$
\left[J_{a}, J_{b}\right]=i \varepsilon_{a b c} J_{c} \quad \text { or }\left[J_{3}, J_{ \pm}\right]= \pm J_{ \pm}, \quad\left[J_{+}, J_{-}\right]=2 J_{3} .
$$

These matrices will be taken to be:

$$
J_{3}=\frac{1}{2}\left(\begin{array}{cccc}
k-1 & 0 & \cdots & 0 \\
0 & k-3 & \cdots & 0 \\
\vdots & \vdots & \vdots & \vdots \\
0 & 0 & \cdots & -(k-1)
\end{array}\right)
$$

and

$$
J_{+}=J_{-}^{*}=J_{-}^{t}=\left(\begin{array}{ccccc}
0 & \sqrt{k-1} & 0 & \cdots & 0 \\
0 & 0 & \sqrt{2(k-2)} & \cdots & 0 \\
\vdots & \vdots & \vdots & \ddots & \vdots \\
0 & 0 & 0 & \cdots & \sqrt{k-1} \\
0 & 0 & 0 & \cdots & 0
\end{array}\right) .
$$

We now make the following ansatz for the solution of Nahm equation:

$$
T_{a}=\frac{J_{a} f_{a}(z)}{i}, \quad a=1,2,3 .
$$

Substituting these in Nahm's equations and using the commutation relations (IV.1) we find that the functions $f_{a}(z)$ satisfy

$$
f_{1}^{\prime}=f_{2} f_{3}, \quad f_{2}^{\prime}=f_{3} f_{1} \text { and } f_{3}^{\prime}=f_{1} f_{2} .
$$

Imposing the boundary conditions, one finds:

$$
f_{1}(z)=\mathbf{K} \frac{d n \mathbf{K} z}{c n \mathbf{K} z}=\frac{\pi \theta_{2} \theta_{3}}{2} \frac{\theta_{3}(z / 2)}{\theta_{2}(z / 2)},
$$

10 We follow the notations and conventions of Erdélyi et. al. [22] 


$$
\begin{aligned}
& f_{2}(z)=\mathbf{K} k^{\prime} \frac{s n \mathbf{K} z}{c n \mathbf{K} z}=\frac{\pi \theta_{3} \theta_{4}}{2} \frac{\theta_{1}(z / 2)}{\theta_{2}(z / 2)}, \\
& f_{3}(z)=\mathbf{K} k^{\prime} \frac{1}{c n \mathbf{K} z}=\frac{\pi \theta_{2} \theta_{4}}{2} \frac{\theta_{4}(z / 2)}{\theta_{2}(z / 2)},
\end{aligned}
$$

where $\operatorname{sn}(\mathbf{K} z)=\operatorname{sn}(\mathbf{K} z, k)$ etc. are the Jacobian elliptic functions and $k^{\prime 2}=1-k^{2} ; k$ being the elliptic modulus ${ }^{11}$ and

$$
\mathbf{K}=\mathbf{K}(k)=\int_{0}^{1} \frac{d x}{\sqrt{\left(1-x^{2}\right)\left(1-k^{2} x^{2}\right)}}
$$

is the complete elliptic integral of the first kind. The theta functions are defined by:

$$
\begin{aligned}
& \theta_{1}(z)=\theta_{1}(z \mid b)=\theta\left[\begin{array}{l}
1 \\
1
\end{array}\right](z)=\sum_{n=-\infty}^{\infty} \exp \left[i \pi\left\{b\left(n+\frac{1}{2}\right)^{2}+2\left(n+\frac{1}{2}\right)\left(z+\frac{1}{2}\right)\right\}\right], \\
& \theta_{2}(z)=\theta_{2}(z \mid b)=\theta\left[\begin{array}{l}
0 \\
1
\end{array}\right](z)=\sum_{n=-\infty}^{\infty} \exp \left[i \pi\left\{b\left(n+\frac{1}{2}\right)^{2}+2\left(n+\frac{1}{2}\right) z\right\}\right], \\
& \theta_{3}(z)=\theta_{3}(z \mid b)=\theta\left[\begin{array}{l}
0 \\
0
\end{array}\right](z)=\sum_{n=-\infty}^{\infty} \exp \left[i \pi\left(b n^{2}+2 n z\right)\right], \\
& \theta_{4}(z)=\theta_{4}(z \mid b)=\theta\left[\begin{array}{l}
1 \\
0
\end{array}\right](z)=\sum_{n=-\infty}^{\infty} \exp \left[i \pi\left\{b n^{2}+2 n\left(z+\frac{1}{2}\right)\right\}\right],
\end{aligned}
$$

and $b=i \mathbf{K}^{\prime} / \mathbf{K}$ and $\mathbf{K}^{\prime}=\mathbf{K}\left(k^{\prime}\right)$ and $\theta_{j}=\theta_{j}(0)=\theta_{j}(0 \mid b)$. In this notation, $\theta_{3}$ is the Riemann theta function defined in Eq. (II.15). Note that these functions are periodic with period 4 and satisfy "conservation laws," corresponding to the Lax form of Nahm's equations:

$$
f_{1}^{2}-f_{2}^{2}=\mathbf{K}^{2}, \quad f_{1}^{2}-f_{3}^{2}=\mathbf{K}^{2} k^{2} \quad \text { and } \quad f_{2}^{2}-f_{3}^{2}=\mathbf{K}^{2} k^{\prime 2} .
$$

Using equations (5) from the introduction, (IV.2) and (IV.3), the matrix $A$ is tridiagonal with $\alpha_{1}, \ldots, \alpha_{k}$ along the diagonal, $\beta_{1}, \ldots, \beta_{k-1}$ above the diagonal and $\gamma_{1}, \ldots, \gamma_{k-1}$ below the diagonal, where:

$$
\begin{aligned}
& \alpha_{j}=-(k+1-2 j) f_{3} \zeta, \\
& \beta_{j}=\sqrt{j(k-j)} \frac{\left(f_{1}+f_{2}\right)+\left(f_{1}-f_{2}\right) \zeta^{2}}{2 i}, \\
& \gamma_{j}=\sqrt{j(k-j)} \frac{\left(f_{1}-f_{2}\right)+\left(f_{1}+f_{2}\right) \zeta^{2}}{2 i} .
\end{aligned}
$$

Thus the curve $S$ is given by $p(\eta, \zeta)=\operatorname{det}(A-\eta I)=0$. Define

$$
a_{2}(\zeta)=-\frac{\mathbf{K}^{2}}{4}\left(1+2\left(k^{2}-k^{\prime 2}\right) \zeta^{2}+\zeta^{4}\right)
$$

\footnotetext{
11 Unfortunately, $k$ sometimes denotes elliptic modulus and sometimes the monopole number. However, the meaning should be clear from the context
} 
Then for the charge $k=2 m$, we have

$$
p(\eta, \zeta)=\prod_{j=1}^{m}\left(\eta^{2}+(2 j-1)^{2} a_{2}(\zeta)\right)
$$

and for $k=2 m+1$,

$$
p(\eta, \zeta)=\eta \prod_{j=1}^{m}\left(\eta^{2}+(2 j)^{2} a_{2}(\zeta)\right)
$$

Alternatively the two equations (IV.11a and b) can be combined as:

$$
p(\eta, \zeta)=\prod_{j=1}^{k}\left(\eta+2 i(k+1-2 j) \sqrt{a_{2}(\zeta)}\right) .
$$

Lemma 4.1. For the elliptic $k$-monopole solutions, Donaldson's rational function is given by:

$$
f(\lambda)=\left(\frac{-1}{\mathbf{K}}\right)^{k-1} \sum_{j=1}^{k} \frac{\left(\begin{array}{c}
k-1 \\
j-1
\end{array}\right)}{\lambda-i(k+1-2 j) \mathbf{K} / 2} .
$$

Proof. Recall (see (III.11)) that Donaldson's rational function is given by:

where

$$
f(\lambda)=w^{t}(\lambda-B)^{-1} w
$$

$$
B=A_{1}(0)=i \mathbf{K} J_{1}
$$

and $w=u(0)$, with

$$
\frac{d u}{d z}+\frac{1}{2} A_{0}(z) u(z)=0, \quad \text { and } \quad u(z) \approx(z-1)^{-(k-1) / 2}(1,0, \ldots, 0)^{t} \quad \text { near } \quad z=1 .
$$

Since the matrix $A_{0}$ is diagonal, this equation can be solved exactly, leading to $w=(-2 / \mathbf{K})^{(k-1) / 2}(1,0, \ldots, 0)^{t}$, and,

$$
f(\lambda)=\left(-\frac{2}{\mathbf{K}}\right)^{k-1}\left[\left(\lambda-i \mathbf{K} J_{1}\right)^{-1}\right]_{11}
$$

Now the matrix $J_{1}$ can be diagonalized by the orthogonal matrix $O=\exp \left[-i(\pi / 2) J_{2}\right]=O^{t}$, i.e. $O$ is the $k$-dimensional representation of $S O(3)$ corresponding to a rotation of $\pi / 2$ about the $x_{2}$ axis, and we find $O^{t} J_{1} O=J_{3}$. To complete the proof note that

$$
\left(v-J_{1}\right)_{11}^{-1}=\left[O\left(v-J_{3}\right)^{-1} O^{t}\right]_{11}=\sum_{j=1}^{k} \frac{\left(O_{1 j}\right)^{2}}{(v-(k+1-2 j) / 2)}
$$

and, using the known representation matrices of $S O(3)$ (see e.g. [25])

$$
\left(O_{1 j}\right)^{2}=\frac{1}{2^{k-1}}\left(\begin{array}{c}
k-1 \\
j-1
\end{array}\right)
$$

We end this section with some remarks. The gauge transformation $C(z)$, is a diagonal matrix, and can be found explicitly and this gives 


$$
Q_{1}=i \mathbf{K} J_{1}, \quad Q_{0}=-2 f_{3}(z) J_{3}, \quad Q_{-1}=\frac{i}{2 \mathbf{K}}\left(\left(f_{1}+f_{2}\right)^{2} J_{+}+\left(f_{1}-f_{2}\right)^{2} J_{-}\right) .
$$

In Sect. II, we have chosen $Q_{1}$ to be diagonal, and this can be done by using the orthogonal matrix $O$ of the previous lemma:

$$
Q_{1}=i \mathbf{K} J_{3}, \quad Q_{0}=-2 f_{3}(z) J_{1}, \quad Q_{-1}=\frac{i}{\mathbf{K}}\left(\left(f_{1}^{2}+f_{2}^{2}\right) J_{3}+2 i f_{1} f_{2} J_{2}\right) .
$$

Also, by taking elliptic modulus $k=0$, these solutions reduce (up to a rotation of the coordinate axes in $\mathbb{R}^{3}$ ) to the axisymmetric solutions of Prasad and Rossi. Secondly, for $k=2$, we can start with a general curve and then impose the condition of Sects. II and III, to show that up to a rotation and translation in $\mathbb{R}^{3}$ that curve must have the form given in (IV.9a), i.e. this gives the most general two monopole solution.

B. Construction of the $k=2$ Solution Using Baker Functions. We now present an example of how one can construct solutions of the Nahm equation using the Baker theory of Sects. I and II, to construct the $k=2$ solution of Sect. IV.A. Even though this does not give any new solutions, besides giving a concrete example, this construction shows that these give all [20] solutions for $k=2$, and, gives an indication of the ingredients that may be necessary for the explicit construction of the general solutions for $k \geqq 3$. We begin by imposing all the constraints on the curve. We then compute the necessary integrals and finally use Eq. (II.21) to construct the $Q$-matrices.

(i) Choice of the curve:

Begin with a general elliptic curve:

$$
\eta^{2}+a_{2}(\zeta)=\eta^{2}+\left(c_{0} \zeta^{4}+c_{1} \zeta^{3}+c_{2} \zeta^{2}+c_{3} \zeta+c_{4}\right)=0
$$

As noted earlier the coefficient of the $\eta^{k-1}=\eta$ term can be chosen to be zero, which corresponds to a choice of origin in $\mathbb{R}^{3}$. The reality condition (II.2b) implies $c_{0}=\bar{c}_{4}, c_{1}=-\bar{c}_{3}$ and $c_{2}=\bar{c}_{2}$. Then using real $(S U(2))$ linear fractional transformations (corresponding to rotations in $\mathbb{R}^{3}$ ) one can choose $c_{1}=c_{3}=0$ and $c_{0}$ to be real and positive to reduce $a_{2}(\zeta)$ to

$$
a_{2}(\zeta)=N^{2}\left(1-2 c \zeta^{2}+\zeta^{4}\right)=N^{2}\left(1+2\left(k^{2}-k^{2}\right) \zeta^{2}+\zeta^{4}\right),
$$

where, one can take $0 \leqq c \leqq \frac{1}{2}$, and with $c=-\cos 2 \alpha=k^{2}-k^{\prime 2}, k=\sin \alpha, k^{\prime}=$ $\sqrt{1-k^{2}}=\cos \alpha$. The branch points, the roots of $a_{2}(\zeta)$, are at $\pm e^{ \pm i \alpha}= \pm k^{\prime} \pm i k$. In this case, we need to impose one $(g=1)$ condition on the curve. To do this, we choose a homology basis of $a$-cycle and $b$-cycle as shown in Fig. 1; then the normalized holomorphic differential is given by:

$$
\omega=\frac{N d \zeta}{\mathbf{K} \eta},
$$

where $\mathbf{K}=\mathbf{K}(k)$ is the complete elliptic integral of the first kind. From Eq. (II.2c), 


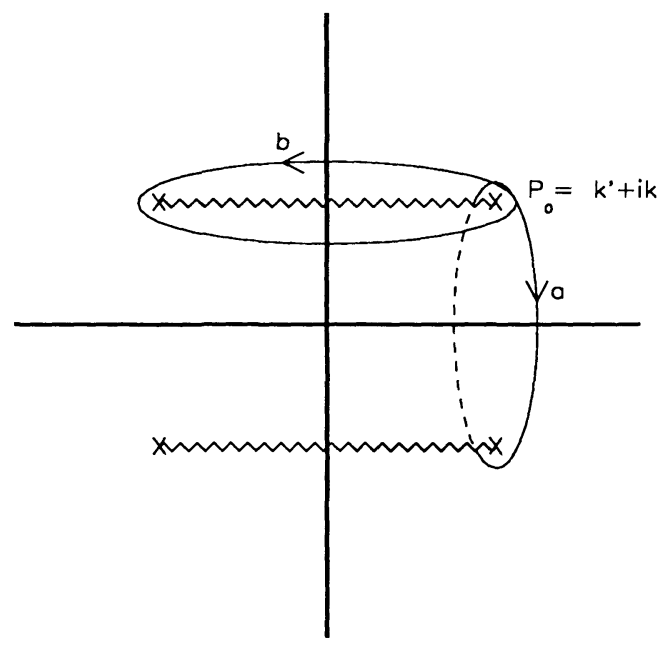

Fig. 1

$\rho_{1}=-i N, \rho_{2}=i N$ and expanding $\omega$ near $\infty_{1}$ and $\infty_{2}$ we find $c^{(1)}=1 /(2 i \mathbf{K})$ and $c^{(2)}=-1 /(2 i \mathbf{K})$. Then, using Theorem II.2,

$$
2\left(\rho_{1} c^{(1)}+\rho_{2} c^{(2)}\right)=\frac{2 N}{\mathbf{K}} \quad \text { is a generator of } \mathbb{Z}^{1} \Rightarrow N= \pm \mathbf{K} / 2 .
$$

Thus, the general elliptic curve (see also $[2,5,20,23,24]$ ) can be taken to be:

$$
\eta^{2}+a_{2}(\zeta)=\eta^{2}+\frac{\mathbf{K}^{2}}{4}\left(1+2\left(k^{2}-k^{\prime 2}\right) \zeta^{2}+\zeta^{4}\right)=0
$$

which is unique up to an $S U(2)$ linear fractional transformation. Of course, the choice of this form of the curve has been dictated by the solution in Sect. IV.A, but any of the standard forms of the curve would serve as well.

(ii) Some differentials, integrals and constants.

With this choice of the curve the normalized holomorphic differential and the Abel map is given by:

$$
\omega=\frac{d \zeta}{4 \eta}, \quad \oint_{a} \omega=1, \quad W(P)=\int_{P_{0}}^{P} \omega,
$$

where $P_{0}$ is chosen to be the branch point $k^{\prime}+i k$. The Riemann "matrix" is:

$$
b=\oint_{b} \omega=i \frac{\mathbf{K}^{\prime}}{\mathbf{K}}, \quad \mathbf{K}^{\prime}=\mathbf{K}\left(k^{\prime}\right) ;
$$

and

$$
\text { Riemann constant }=K=\frac{1+b}{2} .
$$


We also need:

$$
\begin{gathered}
W\left(\infty_{1}\right)=-W\left(\infty_{2}\right)=\frac{1+b}{4}, \\
W\left(0_{1}\right)=-W\left(0_{2}\right)=\frac{1-b}{4} .
\end{gathered}
$$

The above integrals can be evaluated using symmetry with suitable choice of contours. Note that branch through $\infty_{1}$ is defined by $\eta=(-i \mathbf{K} / 2) \sqrt{1+2\left(k^{2}-k^{\prime 2}\right) \zeta^{2}+\zeta^{4}}$.

To construct the meromorphic differentials $\gamma_{\infty}$ and $\gamma_{0}$, recall that $\gamma_{\infty}$ has poles only at $\infty_{j}$ and $\gamma_{\infty}+\gamma_{0}=-d(\eta / \zeta)$. So we have:

$$
\gamma_{\infty}=\frac{\mathbf{K}^{2}\left(\zeta^{2}-\lambda\right)}{4 \eta} \quad \gamma_{0}=\frac{\mathbf{K}^{2}\left(-\zeta^{-2}-\lambda\right)}{4 \eta},
$$

where the constant $\lambda$ is determined by requiring:

$$
\oint_{a} \gamma_{\infty}=\oint_{a} \gamma_{0}=0
$$

and, is given by:

$$
\lambda=\frac{2 \mathbf{E}-\mathbf{K}}{\mathbf{K}}, \text { where } \mathbf{E}=\mathbf{E}(k)=\int_{0}^{1} \frac{\sqrt{1-k^{2} x^{2}}}{\sqrt{1-x^{2}}} d x
$$

is the complete elliptic integral of the second kind.

Using bilinear identities or direct evaluation of the integral, one finds

$$
\oint_{b} \gamma_{\infty}=i \pi=-\oint_{b} \gamma_{0}
$$

Hence from Eq. (II.20):

$$
\kappa=\frac{1}{2} .
$$

Note that one could also have used the "quantization" condition (II.29) to show that the constant $N$ in Eq. (IV.15a) is given by $N= \pm \mathbf{K} / 2$. Also, by direct evaluation of the integral:

$$
v_{1}-v_{2}=\int_{\infty}^{\infty} \gamma_{0}=-2 \int_{P_{0}}^{\infty} \gamma_{0}=-i \frac{\pi}{2}
$$

(iii) The Baker functions and the solution.

First note that $\eta$ and $\zeta$ can be expressed as theta ratios:

$$
\zeta=\frac{\theta\left(W(P)-W\left(0_{1}\right)-\Delta\right) \theta\left(W(P)-W\left(0_{2}\right)-\Delta\right)}{\theta\left(W(P)-W\left(\infty_{1}\right)-\Delta\right) \theta\left(W(P)-W\left(\infty_{2}\right)-\Delta\right)}=-i \frac{\theta_{2}(l) \theta_{4}(l)}{\theta_{1}(l) \theta_{3}(l)},
$$

where, as noted earlier $\theta(u)=\theta_{3}(u)$ is the Riemann theta function and we have used Eqs. (IV.18c and IV.19) and introduced the notation

$$
l=W(P)-W\left(\infty_{1}\right)=W(P)-(1+b) / 4 .
$$


Similarly:

$$
\eta=\frac{i \mathbf{K}}{2} \frac{\theta_{2}^{2} \theta_{4}^{2}}{\theta_{3}} \frac{\theta_{3}(2 l)}{\theta_{1}^{2}(l) \theta_{3}^{2}(l)}=\frac{i \mathbf{K}}{2} \frac{\theta_{2}^{2}}{\theta_{3}^{2}}\left(\frac{\theta_{4}^{2}(l)}{\theta_{1}^{2}(l)}-\frac{\theta_{2}^{2}(l)}{\theta_{3}^{2}(l)}\right) .
$$

To construct the Baker functions, we need to choose the divisor $\delta$ and the functions $g_{j}(P)$. The requirement that $Q_{0}(z)$ has poles at $z= \pm 1$ implies that $\Delta_{1}=0_{2}$ and $\Delta_{2}=0_{1}$. Since the function $g_{1}(P)$ has zeros at $\infty_{2}$ and $\Delta_{1}=0_{2}$ and poles in $\delta=q_{1}+q_{2}$, we can take

$$
g_{1}(P)=\text { constant } \times \frac{\theta_{3}(l) \theta_{3}(l+b / 2)}{\theta_{3}\left(l+l_{1}\right) \theta_{3}\left(l+l_{2}\right)},
$$

$l_{j}=W\left(q_{j}\right)-W\left(\infty_{1}\right)$. Since $g_{1}(P)$ is a function, this must be invariant under $l \rightarrow l+1$ and $l \rightarrow l+b$, which is equivalent to $l_{1}+l_{2}=b / 2$ iff $W(\delta)=-1 / 2$. Using this and $g_{1}\left(\infty_{1}\right)=1$,

$$
g_{1}(P)=\frac{\theta_{3}\left(l_{1}\right) \theta_{2}\left(l_{1}\right)}{\theta_{3} \theta_{2}} \frac{\theta_{3}(l) \theta_{2}(l)}{\theta_{3}\left(l+l_{1}\right) \theta_{2}\left(l-l_{1}\right)}
$$

Similarly,

$$
g_{2}(P)=\frac{\theta_{4}\left(l_{1}\right) \theta_{1}\left(l_{1}\right)}{\theta_{3} \theta_{2}} \frac{\theta_{1}(l) \theta_{4}(l)}{\theta_{3}\left(l+l_{1}\right) \theta_{2}\left(l-l_{1}\right)} .
$$

Then using Eq. (IV.24),

$$
c_{12}=\lim _{P \rightarrow \infty_{2}} \zeta g_{1}(P)=i k^{\prime} \frac{\theta_{3}\left(l_{1}\right) \theta_{2}\left(l_{1}\right)}{\theta_{1}\left(l_{1}\right) \theta_{4}\left(l_{1}\right)}
$$

and

$$
c_{21}=\lim _{P \rightarrow \infty_{1}} \zeta g_{2}(P)=-i k^{\prime} \frac{\theta_{1}\left(l_{1}\right) \theta_{4}\left(l_{1}\right)}{\theta_{3}\left(l_{1}\right) \theta_{2}\left(l_{1}\right)} .
$$

By the symmetry (II.36), $c_{12}=-c_{21}$ requires

$$
\theta_{2}\left(2 l_{1}\right)=0 \Leftrightarrow l_{1}=\frac{1}{4}+\frac{m+n b}{2}, \quad m, n \in \mathbb{Z} .
$$

Note that the form of these equations implies $c_{12}=-c_{21}= \pm i k^{\prime}$. Up to equivalence there are four choices for $l_{1}$ and therefore two choices for the divisor $\delta$, which can be taken to be

$$
l_{1}=\frac{1}{4}, \quad l_{2}=-\frac{1}{4}+\frac{b}{2}, \quad W\left(q_{1}\right)=\frac{1}{2}+\frac{b}{4} \quad \text { and } \quad W\left(q_{2}\right)=\frac{3 b}{4} ; \quad c_{12}=-c_{21}=i k^{\prime}
$$

or

$$
l_{1}=-\frac{1}{4}, \quad l_{2}=\frac{1}{4}+\frac{b}{2}, \quad W\left(q_{1}\right)=\frac{b}{4} \quad \text { and } \quad W\left(q_{2}\right)=\frac{1}{2}+\frac{3 b}{4} ; \quad c_{12}=-c_{21}=-i k^{\prime} .
$$


The Baker functions are

$$
\begin{aligned}
& \Phi_{1}(z, P)=\exp \left[z\left(\int_{P_{0}}^{P} \gamma_{\infty}-v_{1}\right)\right] \frac{\theta_{3}\left(l_{1}\right) \theta_{2}\left(l_{1}\right) \theta_{3}(l) \theta_{2}(l-z / 2)}{\theta_{3} \theta_{2}\left(l+l_{1}\right) \theta_{2}\left(l-l_{1}\right) \theta_{2}(z / 2)} \\
& \Phi_{2}(z, P)=\exp \left[z\left(\int_{P_{0}}^{P} \gamma_{\infty}-v_{1}\right)\right] \frac{\theta_{1}\left(l_{1}\right) \theta_{4}\left(l_{1}\right) \theta_{1}(l) \theta_{4}(l-z / 2)}{\theta_{3} \theta_{2}\left(l+l_{1}\right) \theta_{2}\left(l-l_{1}\right) \theta_{2}(z / 2)}
\end{aligned}
$$

where we have used Eqs. (II.19), (IV.18, 19, 22, 23, 25 and 26) and $\gamma_{\infty}, l_{1}$ are given by Eqs. (IV.20) and (IV.29) respectively.

Finally, since, $\rho_{1}=-\rho_{2}=-i \mathbf{K} / 2$,

$$
Q_{1}=\frac{\mathbf{K}}{2 i}\left(\begin{array}{cc}
1 & 0 \\
0 & -1
\end{array}\right) \quad Q_{0}=i \mathbf{K}\left(\begin{array}{cc}
0 & \alpha_{12} \\
-\alpha_{21} & 0
\end{array}\right)
$$

and using Eq. (II.21) we obtain

$$
\alpha_{12}=-\alpha_{21}=\mp i k^{\prime} e^{-i \pi z / 2} \frac{\theta_{3}\left(\frac{z}{2}+\frac{1}{2}\right) \theta_{3}\left(\frac{b}{2}\right)}{\theta_{3}\left(\frac{1}{2}\right) \theta_{3}\left(\frac{z}{2}+\frac{b}{2}\right)}= \pm \frac{f_{3}(z)}{i \mathbf{K}} .
$$

The choice of the top sign in (IV.32) corresponds to the choice (IV.29b) of $\delta$ and to the solution in Eq. (IV.14), and we can solve for $C(z)$ and obtain $A_{1}(z), A_{0}(z)$ and then take $A_{-1}(z)=-A_{1}^{*}(z)$ to obtain the solution in Sect. IV.A. This calculation is done most easily by diagonalizing $Q_{0}$ by $Q_{a} \rightarrow O^{t} Q_{a} O$ with:

$$
O=O^{t}=O^{-1}=\frac{1}{\sqrt{2}}\left(\begin{array}{cc}
-1 & 1 \\
1 & 1
\end{array}\right)
$$

Then the matrix $C(z)$ is diagonal and given by:

$$
C(z)=C^{-1}(-z)=\left(\begin{array}{cc}
\sqrt{\frac{f_{1}-f_{2}}{\mathbf{K}}} & 0 \\
0 & \sqrt{\frac{f_{1}+f_{2}}{\mathbf{K}}}
\end{array}\right) .
$$

If this construction is carried out with the other choice of sign in Eq. (IV.32); i.e., with $\delta$ given by (IV.29a), one obtains the same $A$ matrices with $z$ replaced by $-z$ or equivalently interchanging $A_{1}$ and $A_{-1}$. Note that these matrices do not satisfy Eqs. (I.1), but that they satisfy appropriately modified (I.1) $(z \rightarrow-z ; \zeta \rightarrow-1 / \bar{\zeta}$ etc.).

Here, we have presented a "first principles" construction of the general two monopole solution. As noted earlier, though this does not give any new solution of Nahm's equations, this gives a concrete example of the procedure of the earlier sections. This procedure can, in principle, be carried out for arbitrary monopole number, however, there are obvious technical difficulties in almost every step of the above construction. 


\section{Conclusion}

We conclude with a summary and some open problems, conjectures and work in progress.

A. Summary. We are interested in the static monopole solutions of the Bogomol'nyi Eq. (1). It has been shown by Hitchin [7] that these solutions are characterized by solutions of Nahm's equations (4) with the conditions $\mathrm{C} 1-\mathrm{C} 4$ or by an algebraic curve satisfying the conditions (II.23). Our primary focus has been the explicit solution of Nahm's equations. Note that we are using a slightly modified form of Hitchin's boundary conditions.

In Sect. I there is a gauge transformation $Q_{\alpha}(z)=C^{-1}(z) A_{\alpha}(z) C(z), \alpha=0, \pm 1$ of the Lax form of Nahm's Eq. (I.1). In Theorem I.1, the boundary conditions are reformulated in terms of the linear operator $D+\frac{1}{2} Q_{0}$.

In Theorem II.1 we characterize the eigenfunctions of the operator $D+Q_{+}$ and then present explicit formulae for the Baker functions and the $Q_{0}$ matrix in terms of the meromorphic differential $\gamma_{\infty}$ and Riemann theta functions. The gauge invariant boundary conditions require that the curve must satisfy the $g=(k-1)^{2}$ conditions given in Eq. (II.29). These are transcendental conditions involving loop integrals of the meromorphic differential $\gamma_{\infty}$. Theorem II.2 provides an alternative formulation of these conditions in terms of normalized holomorphic differentials.

Section III is devoted to the proof of Theorem I.1 and the relation of Donaldson's rational function to the Baker data.

In Sect. IV.A, we present a class of elliptic solutions of Nahm's equations for arbitrary monopole number, which correspond to singular (for $k \geqq 3$ ) curves with elliptic components. Donaldson's rational function for these solutions has a simple partial fractions expansion. In Sect. IV.B we provide an example $(k=2)$ of the following "algorithm" for the construction of the solutions of Nahm's equations:

1. Start with an arbitrary curve of the form (II.2a), satisfying the reality conditions (II.2b). Then impose the "quantization" conditions (II.29) or equivalently the conditions in Theorem II.2.

2. Construct the Baker functions and the matrices $Q_{1}, Q_{0}$ using Eqs. (II.19) or (II.35) and (II.1, 2c, 2d, 10 and 21). This requires the function $g_{j}(P)$ and its divisor which involves $\delta$ and $\Delta_{j}$; the divisor can be determined using the requirements that the Baker functions $\Phi_{j}(z, P)$ have poles at $z= \pm 1$ (see Eq. (II.33)) and the skew-symmetry conditions $c_{i j}=-c_{j i}$.

3. Solve the linear equation $C^{\prime}=\frac{1}{2} Q_{0} C, C(0)=I$ for the gauge transformation. Then construct $A_{0}, A_{1}$ using (I.10a) and $A_{-1}=A_{1}^{*}$.

We note that, as in the two monopole case there may be two sets of $A$ matrices, one set satisfying the original Nahm's equations and the other set satisfying the equations given by $z \rightarrow-z$ in the Nahm's equations.

B. Some Open Problems and Conjectures. A fundamental open problem is the existence (and also the construction) of non-singular curves (for $k>2$ ) satisfying the transcendental Eq. (II.29). We believe that the answer is affirmative, and 
hope to study this question at least perturbatively for the three monopole solutions.

Another open problem is to extend the Baker theory to singular curves, which may arise in some limiting case as in Sect. IV.A. We have some partial results on this and further work is in progress.

The Nahm matrices can be expressed in terms of simple exponentials times a ratio of theta functions and are periodic in $z$ with period $=4$. The proof of periodicity requires showing $v_{i}-v_{j}=i \pi n / 2$. We have expressed $Q_{1}, Q_{0}$ in terms of theta functions; however, to show this for the $A$ matrices may involve some cancellations in the gauge transformation matrix $C(z)$. An alternative approach to this question is to use the "squared Baker functions" [12]. A discussion of this is deferred to a future publication.

One would also like to solve the linear Eq. (8) of the introduction and construct the solution of the Bogomol'nyi equations given by (9). Recall that the zeros of the Higg's field $\Phi\left(x_{1}, x_{2}, x_{3}\right)$ give the "positions" of the monopoles, and are important in the study of monopole dynamics. The explicit formulas presented here may be useful for this purpose.

Finally, as noted in the introduction, we would like to see how our study may be used to better understand and extend the results of Atiyah and Hitchin on the dynamics of slowly moving monopoles.

Acknowledgements. We thank H. Flaschka for suggesting the reduction of Nahm's equations used in Sect. I, and, a very careful reading of the manuscript.

\section{References}

1. Bogomol'nyi, E. B.: Yad. Fiz. 24, 861-870, (1976) [Sov. J. Nucl. Phys. 24, 449 (1976)]; Prasad, M. K., Sommerfeld, C. M.: Phys. Rev. Lett. 35, 760 (1975)

2. Nahm, W.: All self-dual multimonopoles for arbitrary gauge groups. (preprint), TH.3172-CERN (1981)

3. Atiyah, M., Hitchin, N. J.: Geometry and dynamics of magnetic monopoles. Princeton, NJ: Princeton University Press 1988

4. Donaldson, S. K.: Commun. Math. Phys. 96, 387-407 (1984)

5. See e.g. Monopoles in Quantum Field Theory. Craigie, N. S., Goddard, P., Nahm, W. (eds.). Singapore: World Scientific 1982. For an alternative method of solving the Bogomol'nyi equations based on the Atiyah-Ward (Commun. Math. Phys. 55, 117-124 (1977)) approach see Ward, R. S.: Commun. Math. Phys. 79, 317-325 (1981); Prasad, M. K.: Commun. Math. Phys. 80, 137-149 (1981); Prasad, M. K., Sinha, A., Chau-Wang, L-L.: Phys. Rev. D23, 2321-2334 (1981); Prasad, M. K., Rossi, P. [20]; Ward, R. S.: Phys. Lett. 102B, 136-138 (1981); Ward, R. S.: Commun. Math. Phys. 80, 563-574 (1981); Corrigan, E., Goddard, P.: Commun. Math. Phys. 80, 575-587 (1981). See also Forgás, P., Horváth, Z., Palla, L.: Phys. Lett. 102B, 131-135 (1981) and Phys. Lett. 109B, 200-204 (1982)

6. Hitchin, N. J.: Commun. Math. Phys. 83, 579-602 (1982)

7. Hitchin, N. J.: Commun. Math. Phys. 89, 145-190 (1983)

8. Atiyah, M., Hitchin, N., Drinfel'd, V., Manin, Yu.: Phys. Lett. 65A, 185 (1978)

9. Baker, H. S.: Abel's Theorem and the Allied Theory. Cambridge: Cambridge University Press 1897

10. Krichever, I. M.: Funct. Anal. Appl. 11, 144-146 (1977)

11. Griffiths, P. A.: Am. J. Math. 107, 1445-1484 (1985)

12. Flaschka, H., Newell, A. C., Ratiu, T.: Physica 9D, 300-323 (1983)

13. Flaschka, H., Forest, M. G., McLaughlin, D. W.: Commun. Pure Appl. Math. 33, 739-784 (1980)

14. Lax, P., Levermore, D.: Commun. Pure Appl. Math. 33, 739-784 (1980) 
15. Ercolani, N., Forest, M. G., Montgomery, R., McLaughlin, D. W.: Duke Math. J. 55, 949-983 (1987)

16. Date, E.: Osaka J. Math. 19, 125-158 (1982)

17. For background information on Algebraic Geometry and Riemann Surface Theory see e.g. Griffiths, P., Harris, J.: Principles of algebraic geometry. New York: Wiley 1978; and Springer, G.: Introduction to Riemann surfaces. Reading, MA: Addison-Wesley 1957

18. Siegel, C. L.: Topics in complex function theory. Vol. II. New York: Wiley 1969

19. Hartshorne, R.: Commun. Math. Phys. 59, 1-15 (1978)

20. Hurtubise, J.: Commun. Math. Phys. 92, 195-202 (1983)

21. Fay, J. D.: Theta functions on Riemann surfaces. Lecture Notes in Mathematics, vol. 352. Berlin, Heidelberg, New York: Springer 1973

22. Erdélyi, A et. al.: Higher transcendental functions, Bateman manuscript project, vol. 2. New York: McGraw-Hill (1953)

23. Prasad, M. K., Rossi, P.: Phys. Rev. Lett. 46, 806 (1981); Phys. Rev. D24, 2182 (1981)

24. Brown, S. A., Panagopoulos, H., Prasad, M. K.: Phys. Rev. D26, 854 (1982)

25. Bidenharn, L. C., Louck, J. D.: Angular momentum in quantum physics theory and application. Reading, MA: Addison-Wesley 1981

Communicated by A. Jaffe

Received October 13, 1988; in revised form April 14, 1989 